\title{
A classification of semilocal vortices in a Chern-Simons theory
}

\author{
Jann-Long Chern *, Zhi-You Chen, Sze-Guang Yang \\ Department of Mathematics, National Central University, Chung-Li 32001, Taiwan
}

Received 5 October 2011; received in revised form 29 August 2013; accepted 25 November 2014

Available online 3 December 2014

\begin{abstract}
We consider a Chern-Simons theory of planar matter fields interacting with the Chern-Simons gauge field in a SU $(N)_{\text {global }} \otimes$ $\mathrm{U}(1)_{\text {local }}$ invariant fashion. We classify the radially symmetric soliton solutions of the system in terms of the prescribed value of magnetic flux associated with this model. We also prove the uniqueness of the topological solution in a certain condition.
\end{abstract}

๑ 2014 L'Association Publications de l'Institut Henri Poincaré. Published by Elsevier B.V. All rights reserved.

\section{Résumé}

Nous étudions une théorie Chern-Simons de champs de matière plans interagissant avec le champ de jauge de Chern-Simons d'une manière invariante par le groupe $\mathrm{SU}(N)_{\text {global }} \otimes \mathrm{U}(1)_{\text {local. }}$. Nous classons les solutions solitons radialement symétriques du système en fonction de la valeur prescrite d'un flux magnétique associé à ce modèle. Nous prouvons également l'unicité de la solution topologique sous une certaine condition.

๑ 2014 L'Association Publications de l'Institut Henri Poincaré. Published by Elsevier B.V. All rights reserved.

MSC: 35J60; 35J55

Keywords: Chern-Simons-Higgs model; Classification of nontopological solutions for elliptic system; Uniqueness result of topological solutions

\section{Introduction}

The theory of semilocal vortices has a global $\mathrm{SU}(N)$ symmetry in addition to the local $\mathrm{U}(1)$ symmetry, $\mathrm{SU}(N)_{\text {global }} \otimes \mathrm{U}(1)_{\text {local }}$, which is described by the Lagrangian:

$$
\mathcal{L}=\frac{\kappa}{4} \epsilon^{\mu \nu \alpha} F_{\mu \nu} A_{\alpha}+\left(D_{\mu} \phi\right)^{\dagger}\left(D^{\mu} \phi\right)-\frac{1}{\kappa^{2}}|\phi|^{2}\left(|\phi|^{2}-1\right)^{2}
$$

\footnotetext{
Work supported by Ministry of Science and Technology, Taiwan (grants NSC101-2115-M-008-009-MY3 and MOST103-2115M-008-011-MY3).

* Corresponding author.

E-mail addresses: chern@math.ncu.edu.tw (J.-L.Chern), zhiyou@ math.ncu.edu.tw (Z.-Y. Chen), sgyang@ math.ncu.edu.tw (S.-G. Yang).
} 
where $A_{\mu}, \mu=0,1,2$, is the gauge field on the $(2+1)$-dimensional space $\mathbb{R}^{2,1}$ of metric tensor $\left(g_{\mu \nu}\right)=$ $\operatorname{diag}(1,-1,-1), F_{\mu \nu}=\left(\partial / \partial x^{\mu}\right) A_{\nu}-\left(\partial / \partial x^{\nu}\right) A_{\mu}$ is the corresponding gauge curvature tensor, $D_{\mu}=\left(\partial / \partial x^{\mu}\right)-\mathrm{i} A_{\mu}$ is the gauge covariant derivative, $\phi=\left(\phi_{1}, \phi_{2}, \ldots, \phi_{K}\right)$ is $K$-component scalar field with $\phi^{\dagger}=\left(\phi_{1}^{*}, \phi_{2}^{*}, \ldots, \phi_{K}^{*}\right)$ (here $*$ denotes complex conjugation), $\epsilon_{\mu \nu \alpha}$ is the totally skew-symmetric tensor with $\epsilon_{012}=1$, and $\kappa>0$ is the Chern-Simons coupling constant. We mention that there are more interesting background and further studies of the corresponding Abelian Higgs model with $\mathrm{SU}(N)_{\text {global }} \otimes \mathrm{U}(1)_{\text {local }}$ symmetry, e.g., in Refs. [2,7,8] and [13].

In the static case, when the Bogomol'nyi bound is saturated, we deduce the following self-dual equations:

$$
\left\{\begin{array}{l}
\left(D_{1} \pm \mathrm{i} D_{2}\right) \phi_{j}=0, \quad j=1, \ldots, K, \\
F_{12} \pm \frac{2}{\kappa^{2}}|\phi|^{2}\left(|\phi|^{2}-1\right)=0 .
\end{array}\right.
$$

Without loss of generality, we will only take the upper (plus) sign into account. The flux corresponding to the magnetic field $F_{12}$ is given by

$$
\Phi=\int_{\mathbb{R}^{2}} F_{12} d \mathbf{x}
$$

The system (1) is associated with one of the following boundary conditions that either

(i) $|\phi(\mathbf{x})| \rightarrow 1$ as $|\mathbf{x}| \rightarrow+\infty$, or

(ii) $|\phi(\mathbf{x})| \rightarrow 0$ as $|\mathbf{x}| \rightarrow+\infty$.

The first is called topological boundary condition and the second is nontopological. The point vortices of the system are identified with zeros of $\phi_{j}, j=1, \ldots, K$. We remark that the existence of the topological as well as nontopological (multi-vortex) solutions has been proved by Chae in [2]. In the present article we set forth a classification of the radially symmetric solutions to the system.

We locate the zeros of $\phi_{j}$ at the origin with the multiplicities $N_{j}$ (positive integers) for $j=1, \ldots, K$. For simplicity, we set $\kappa=2$. With the substitution $u_{j}=\log \left|\phi_{j}\right|^{2}, j=1, \ldots, K$, or equivalently

$$
\phi_{j}(z)=\exp \left[\frac{u_{j}}{2}+\mathrm{i} N_{j} \operatorname{Arg}(z)\right], \quad z=x_{1}+\mathrm{i} x_{2},\left(x_{1}, x_{2}\right)=\mathbf{x} \in \mathbb{R}^{2},
$$

the system (1) is reduced to the following semilinear elliptic system:

$$
\left\{\begin{array}{l}
\Delta u_{j}=\left(\sum_{k=1}^{K} e^{u_{k}}\right)\left(\sum_{k=1}^{K} e^{u_{k}}-1\right)+4 \pi N_{j} \delta_{0} \quad \text { in } \mathbb{R}^{2}, j=1, \ldots, K, \\
\beta=\beta(\mathbf{u})=\frac{1}{2 \pi} \int_{\mathbb{R}^{2}}\left(\sum_{k=1}^{K} e^{u_{k}}\right)\left(1-\sum_{k=1}^{K} e^{u_{k}}\right) d \mathbf{x} \in(0,+\infty),
\end{array}\right.
$$

where $\mathbf{u}=\left(u_{1}, \ldots, u_{K}\right), \Delta \equiv \frac{\partial^{2}}{\partial x_{1}^{2}}+\frac{\partial^{2}}{\partial x_{2}^{2}}$ and $\delta_{0}$ denotes the Dirac delta function with point mass at the origin. Note that $\Phi=\pi \beta$ by definition. The topological and nontopological boundary conditions (i) and (ii) above are equivalent respectively to the following statements:

$$
\begin{aligned}
& e^{u_{j}} \rightarrow \sigma_{j} \quad \text { as }|\mathbf{x}| \rightarrow+\infty \text { for } j=1, \ldots, K \text { with } \sigma_{j} \geq 0 \text { and } \sum_{j=1}^{K} \sigma_{j}=1 ; \\
& e^{u_{j}} \rightarrow 0 \quad \text { as }|\mathbf{x}| \rightarrow+\infty \text { for } j=1, \ldots, K .
\end{aligned}
$$

For simplicity, through a rearrangement we may assume that $N_{1} \geq N_{2} \geq \cdots \geq N_{K} \geq 0$, and for purely mathematical sake, that each $N_{j}$ is not necessarily an integer. Our first consequence is stated as follows. 
Theorem 1.1. Let $\mathbf{u}(r)=\left(u_{1}, \ldots, u_{K}\right)(r)$ be a radially symmetric solution of $E q$. (2) with $r=|\mathbf{x}|$, and let

$$
\alpha_{1}=\max \left\{2\left(N_{1}+1\right), 4\left(N_{K}+1\right)\right\}, \quad \alpha_{2}=\max \left\{4\left(N_{1}-N_{K}\right), 4\left(N_{K}+1\right)\right\} .
$$

Then $\beta \in E:=\left\{2 N_{1}\right\} \cup\left(\alpha_{1},+\infty\right)$. Conversely, for a prescribed $\beta \in E$, the following statements are true:

(a) If $\beta=2 N_{1}$, then $\mathbf{u}$ is topological and can be characterized by

$$
u_{j}(r)=v(r)+2 N_{j} \log r+\log c_{j}, \quad c_{j}>0, j=1, \ldots, K,
$$

where $v(|\mathbf{x}|) \in C_{\mathrm{loc}}^{2}\left(\mathbb{R}^{2}\right)$ is a solution of

$$
\left\{\begin{array}{l}
v^{\prime \prime}+\frac{1}{r} v^{\prime}=f e^{v}\left(f e^{v}-1\right), \quad f(r)=\sum_{j=1}^{K} c_{j} r^{2 N_{j}}, \quad r>0, \\
v(r)=-2 N_{1} \log r+O(1) \quad \text { as } r \rightarrow+\infty .
\end{array}\right.
$$

(b) If $\beta \in\left(\alpha_{2},+\infty\right)$ then $\mathbf{u}$ is nontopological and can be characterized by

$$
u_{j}(r)=v(r)+2 N_{j} \log r+\log c_{j}, \quad c_{j}>0, j=1, \ldots, K,
$$

where $v(|\mathbf{x}|) \in C_{\text {loc }}^{2}\left(\mathbb{R}^{2}\right)$ is a solution of

$$
\left\{\begin{array}{l}
v^{\prime \prime}+\frac{1}{r} v^{\prime}=f e^{v}\left(f e^{v}-1\right), \quad f(r)=\sum_{j=1}^{K} c_{j} r^{2 N_{j}}, \quad r>0, \\
v(r)=-\beta \log r+O(1) \quad \text { as } r \rightarrow+\infty .
\end{array}\right.
$$

Moreover, $\alpha_{1}=\alpha_{2}=4 N_{K}+4$ when $N_{1} \leq 2 N_{K}+1$.

(c) If $N_{1}>2 N_{K}+1$, then there exists a constant $\alpha_{3}=\alpha_{3}\left(N_{1}, \ldots, N_{K}\right) \in\left(\alpha_{1}, \alpha_{2}\right)$ such that (2) possesses a nontopological solution $\left(u_{1}, \ldots, u_{K}\right)$ which is characterized in $(b)$ for each $\beta \in\left[\alpha_{3}, \infty\right)$. Moreover, $\beta(\mathbf{u}) \in\left[\alpha_{3}, \infty\right)$ for any nontopological solution $\mathbf{u}=\left(u_{1}, \ldots, u_{K}\right)$ of $(2)$.

Remark 1.1. As shown in Appendix A, each solution of (2) satisfying the (topological) boundary condition (3) is automatically radially symmetric. So that the radial symmetry premise can be removed from Theorem 1.1 for the topological solutions.

Remark 1.2. In the case $N_{1}>2 N_{K}+1$, it turns out that $\alpha_{1}=2\left(N_{1}+1\right)$ and $\alpha_{2}=4\left(N_{1}-N_{K}\right)$ with $\alpha_{1}<\alpha_{2}$.

Remark 1.3. When $K=1$, the semilocal system (2) is reduced to the well-known Abelian Chern-Simons Higgs model

$$
\left\{\begin{array}{l}
\Delta u=e^{u}\left(e^{u}-1\right)+4 \pi N \delta_{0} \quad \text { in } \mathbb{R}^{2} \\
\beta=\beta(u)=\frac{1}{2 \pi} \int_{\mathbb{R}^{2}} e^{u}\left(1-e^{u}\right) d \mathbf{x} \in(0,+\infty)
\end{array}\right.
$$

in which the sufficient and necessary condition for Eq. (7) possessing a radially symmetric solution is that $\beta \in\{2 N\} \cup$ $(4 N+4,+\infty)$. This specializes the consequence of Theorem 1.1.

In the case $K=1$ we remark that radial solutions of (7) depend uniquely on the values of $\beta$; please see e.g. [5] and [4] for the such uniqueness results. In the case $K \geq 2$, there can be two solutions $\mathbf{u}_{1} \neq \mathbf{u}_{2}$ such that $\beta\left(\mathbf{u}_{1}\right)=\beta\left(\mathbf{u}_{2}\right)$. In fact, as shown at the beginning of Section 2, any solution $\mathbf{u}=\left(u_{1}, \ldots, u_{K}\right)$ of Eq. (2) can be written as

$$
u_{j}(r)=v(r)+2 N_{j} \log r+\log c_{j}, \quad c_{j}>0, j=1, \ldots, K,
$$

where $v=v\left(\beta, c_{1}, \ldots, c_{K}\right)$ satisfies the equation 


$$
\left\{\begin{array}{l}
v^{\prime \prime}+\frac{1}{r} v^{\prime}=f e^{v}\left(f e^{v}-1\right), \quad f(r)=\sum_{j=1}^{K} c_{j} r^{2 N_{j}}, \quad r>0, \\
v(r)=-\beta \log r+O(1) \quad \text { as } r \rightarrow+\infty .
\end{array}\right.
$$

In other words, $\mathbf{u}$ depends on $\beta$ and the $K$-tuple parameter $\tau=\left(c_{1}, \ldots, c_{K}\right)$. Let $\beta$ be fixed. Assume that $v=$ $v\left(c_{1}, \ldots, c_{K}\right)$ and $\tilde{v}=v\left(\tilde{c}_{1}, \ldots, \tilde{c}_{K}\right)$ are solutions of (8) corresponding parameters $\left(c_{j}\right)_{j=1}^{K}$ and $\left(\tilde{c}_{j}\right)_{j=1}^{K}$ respectively. So we have two solutions $\mathbf{u}$, $\tilde{\mathbf{u}}$ of (2) given by $u_{j}(r)=v(r)+2 N_{j} \log r+\log c_{j}$ and $\tilde{u}_{j}(r)=\tilde{v}(r)+2 N_{j} \log r+\log \tilde{c}_{j}$, $j=1, \ldots, K$. If $\mathbf{u}=\tilde{\mathbf{u}}$, then there must be a constant $\lambda>0$ such that

$$
\log \frac{c_{j}}{\tilde{c}_{j}}=v-\tilde{v}=\lambda, \quad j=1,2, \ldots, K .
$$

Consequently, we may select two different parameters $\left(c_{j}\right)_{j=1}^{K},\left(\tilde{c}_{j}\right)_{j=1}^{K}$ on the spherical region

$$
\Sigma=\left\{\left(c_{1}, \ldots, c_{K}\right): c_{1}^{2}+\cdots+c_{K}^{2}=1 \text { with } c_{1}, \ldots, c_{K}>0\right\},
$$

so that $\mathbf{u} \neq \tilde{\mathbf{u}}$. Although a solution of (2) cannot be uniquely determined by any prescribed $\beta$ in the case $K \geq 2$, the following theorem shows that a solution of (5) is unique under a certain condition. Such a result represents some kind of uniqueness for the model. We state the theorem as follows:

Theorem 1.2. If $N_{1}-N_{K} \leq 1$, then for any given $c_{j}>0, j=1, \ldots, K$, the solution of (5) is unique. Moreover, the topological solution of (2), which is denoted by $\mathbf{u}_{\tau, 2 N_{1}}=\mathbf{u}_{\tau}$, is uniquely determined by the parameter $\tau$ belonging to the region $\Sigma$ given in (9).

By the way, we remark that Theorem 1.2 can be equivalently characterized from an alternative viewpoint by means of the initial value at the origin. Since all topological solutions of (2) are radially symmetric with respect to the origin as we have shown in Appendix A of the present paper, we may rewrite the system (2) as the following system associated the initial value at the origin:

$$
u_{j}^{\prime \prime}(r)+\frac{1}{r} u_{j}^{\prime}(r)+\left(\sum_{j=1}^{K} e^{u_{j}}\right)\left(1-\sum_{j=1}^{K} e^{u_{j}}\right)=0, \quad r>0,
$$

with behavior at the origin that

$$
u_{j}(r)=2 N_{j} \log r+\rho_{j}+o(1) \quad \text { as } r \rightarrow 0^{+} .
$$

According the uniqueness result in Theorem 1.2, we obtain the following assertion about the structure of topological solutions of (10) in terms of $\left(\rho_{1}, \cdots, \rho_{K}\right)$.

Corollary 1.3. Suppose that $N_{1}-N_{K} \leq 1$. Then for any $\ell=\left(\ell_{1}, \ldots, \ell_{K-1}\right) \in \mathbb{R}^{K-1}$, there exists a unique $\left(\rho_{1}^{*}(\ell), \ldots, \rho_{K}^{*}(\ell)\right) \in \mathbb{R}^{K}$ such that $\left(u_{1}\left(\rho_{1}^{*}, \ldots, \rho_{K}^{*}\right), \ldots, u_{K}\left(\rho_{1}^{*}, \ldots, \rho_{K}^{*}\right)\right)$ is a topological solution of $(10)$ with $\rho_{j}^{*}(\ell)-\rho_{1}^{*}(\ell)=\ell_{j-1}$ for $j=2, \ldots, K$.

The paper is organized as follows. In Section 2 we show that the solutions of (2) can be reduced to a single profile equation, and furthermore make an analysis on its solution structure. In addition, we carry out a uniqueness result under a certain condition. In Sections 3 and 4 we exhibit the existence of the nontopological solutions with respect to prescribed values of magnetic flux, in order to detect an optimal lower bound of the possible values. Finally, in Appendix A of the present paper, we include a proof about the radial symmetry for the topological solution of the system (2).

\section{Analysis of the solution structure}

In view of the $K$-component system (2), we see that $\Delta\left(u_{i}-u_{j}\right)=4 \pi\left(N_{i}-N_{j}\right) \delta_{0}$ for any $i, j \in\{1, \ldots, K\}$, which indicates that 


$$
u_{i}(r)=u_{j}(r)+2\left(N_{i}-N_{j}\right) \log r+h_{i j}(r),
$$

where $h_{i j}$ is a harmonic function. Since $\beta<+\infty$, we have $\lim _{r \rightarrow \infty} r u_{i}^{\prime}(r)=2 N_{i}-\beta$ for $i=1, \ldots, K$ and it is possible to extract a positive constant $C$ such that

$$
\left|u_{1}(r)\right|+\cdots+\left|u_{K}(r)\right| \leq C \log (1+r), \quad r>0 .
$$

It follows that $h_{i j}$ is in fact a constant. For the sake, through a simple substitution that

$$
u_{j}(r)=v(r)+\log c_{j}+2 N_{j} \log r,
$$

where $c_{j}>0$ for $j=1, \ldots, K$, we obtain a further reduction of the system (2), namely

$$
\left\{\begin{array}{l}
v^{\prime \prime}+\frac{1}{r} v^{\prime}=f e^{v}\left(f e^{v}-1\right), \quad r>0, \\
\beta=\int_{0}^{\infty} r f e^{v}\left(1-f e^{v}\right) d r \in(0,+\infty),
\end{array}\right.
$$

where $f(r)=\sum_{j=1}^{K} c_{j} r^{2 N_{j}}$ with $N_{1} \geq N_{2} \geq \cdots \geq N_{K} \geq 0$. We set off a structure analysis for the solutions of (13). First of all, for any given $K$-tuple $\left(c_{1}, c_{2}, \ldots, c_{K}\right)$ with $c_{j}>0$, we consider the solution $v(r)=v(r ; s)$ solving the initial value problem

$$
\left\{\begin{array}{l}
v^{\prime \prime}+\frac{1}{r} v^{\prime}=f e^{v}\left(f e^{v}-1\right), \\
v(0)=s, \quad v^{\prime}(0)=0, \quad s \in \mathbb{R} .
\end{array}\right.
$$

The solution of (14) can be classified into three types; in fact, we have:

Theorem 2.1. Let $v$ be a solution of (14). Then $v$ belongs to one of the following classes given in the sense that

0-Type: $\quad f(r) e^{v(r)} \rightarrow 0 \quad$ as $r \rightarrow+\infty$;

1-Type: $\quad f(r) e^{v(r)} \rightarrow 1 \quad$ as $r \rightarrow+\infty$;

$\infty$-Type: $\quad v(r)$ blows up at a finite $r$.

Before proving Theorem 2.1, we introduce properties that $2 N_{K} \leq r f^{\prime} / f \leq 2 N_{1}$ and that

$$
\left(\frac{r f^{\prime}}{f}\right)^{\prime}(r)=\frac{1}{f^{2}(r)} \sum_{i<j} 4 N_{i}\left(N_{i}-N_{j}\right) c_{i} c_{j} r^{2\left(N_{i}+N_{j}\right)-1} \geq 0 .
$$

They can be obtained by simple calculations. Note that the properties are independent of the choice of $\left(c_{1}, c_{2}, \ldots, c_{K}\right)$. So in the following content we assume $c_{1}=c_{2}=\cdots=c_{K}=1$ for simplicity.

Lemma 2.1. Let $v$ solve the problem (14). Then the function $f e^{v}-1$ cannot attain a nonnegative local maximum at any finite $r>0$.

Proof. Assume $g=f e^{v}-1$ has a nonnegative local maximum at $r=r_{1}$. Then $g^{\prime}\left(r_{1}\right)=0$ and there is a small $\epsilon>0$ such that $\left(r v^{\prime}\right)^{\prime} \geq 0$ on $\left[r_{1}, r_{1}+\epsilon\right)$. Note that

$$
g^{\prime}(r)=\frac{f e^{v}}{r} h(r), \quad \text { where } h(r)=\frac{r f^{\prime}}{f}+r v^{\prime} .
$$

Hence $h\left(r_{1}\right)=r_{1} g^{\prime}\left(r_{1}\right) / f\left(r_{1}\right) e^{v\left(r_{1}\right)}=0$, and furthermore,

$$
h^{\prime}(r)=\left(\frac{r f^{\prime}}{f}\right)^{\prime}+\left(r v^{\prime}\right)^{\prime} \geq 0
$$

on $\left[r_{1}, r_{1}+\epsilon\right)$. It follows that $h(r) \geq 0$ on $\left[r_{1}, r_{1}+\epsilon\right)$. Therefore, $g^{\prime}(r) \geq 0$ on $\left[r_{1}, r_{1}+\epsilon\right)$. This contradicts the assumption that $g\left(r_{1}\right)$ is a local maximum. 
Lemma 2.2. Let $u_{1}(r)=2 N_{1} \log r+v(r)$ where $v$ solves $(14)$ in an interval $I=[0, \omega), \omega \in \mathbb{R} \cup\{\infty\}$. If $u_{1}^{\prime}\left(r_{1}\right)<0$ for some $r_{1} \in I$, then $v$ must be of the 0-Type.

Proof. It suffices to show that $f e^{v}<1$ for any $r$ as far as $v(r)$ is defined. In fact, since $u_{1}^{\prime}$ is positive near $r=0$ and negative at $r=r_{1}$, the function $u_{1}$ attains its maximum $\sup _{0<r<r_{1}} u_{1}(r)$ at some locus $r_{0} \in\left(0, r_{1}\right)$. From (14),

$$
2 N_{1}-\int_{0}^{r_{1}} r f e^{v}\left(1-f e^{v}\right) d r=r_{1} u_{1}^{\prime}\left(r_{1}\right)<0 .
$$

Applying the fact $u_{1}^{\prime}\left(r_{0}\right)=0$ and using $r_{0}$ in substitution for $r_{1}$ in the equality (17), we see that the derived integral over $\left(0, r_{0}\right)$ has value $2 N_{1}$; this indicates

$$
\int_{r_{0}}^{r_{1}} r f e^{v}\left(1-f e^{v}\right) d r>0
$$

We conclude $f e^{v}<1$ in $\left(0, r_{1}\right)$, because, by Lemma 2.1, once $f\left(r_{0}\right) e^{v\left(r_{0}\right)} \geq 1$, the function $f(r) e^{v(r)}-1$ is positive for $r>r_{0}$ and thus (18) cannot happen. Assume $f(\bar{r}) e^{v(\bar{r})}=1$ for some $\bar{r} \geq r_{1}$ with the property $f(r) e^{v(r)}<1$ for $r \in(0, \bar{r})$ and $f(r) e^{v(r)}>1$ for $r>\bar{r}$. Then $u_{1}^{\prime}(r)<0$ whenever $r_{0}<r<\bar{r}$ and therefore $f e^{v}$ is strictly decreasing on the interval $\left(r_{0}, \bar{r}\right)$. Since $f e^{v}=1$ at $r=\bar{r}$ in our assumption, we come to the conclusion that $f\left(r_{0}\right) e^{v\left(r_{0}\right)}>1$, a contradiction.

Proof of Theorem 2.1. From Lemma 2.1, if $f\left(r_{0}\right) e^{v\left(r_{0}\right)} \geq 1$ for some $r_{0}$, then $f e^{v}$ is increasing in the interval $\left(r_{0},+\infty\right)$ and then $\Delta v \geq \lambda f e^{v}$ for $r \geq r_{0}$ where $\lambda$ is a positive constant. Hence $v$ blows up at a finite $r$ (cf. e.g. [12]). Otherwise, $v$ is an entire solution of (14) satisfying $f e^{v}<1$ all the time; in the meanwhile $v$ is a decreasing function which approaches to $-\infty$ as $r \rightarrow+\infty$. Note that in the last case we have

$$
\beta=\int_{0}^{\infty} r f e^{v}\left(1-f e^{v}\right) d r<+\infty
$$

For, if the integral $\beta=+\infty$, it is possible to select $r_{1}>0$ such that $r_{1} v^{\prime}\left(r_{1}\right) \leq-4 N_{1}-4$. By Eq. (14),

$$
r v^{\prime}(r)=r_{1} v^{\prime}\left(r_{1}\right)-\int_{r_{1}}^{r} r f e^{v}\left(1-f e^{v}\right) d r \leq-4 N_{1}-4, \quad r \geq r_{1},
$$

implying $v(r) \leq-\left(4 N_{1}+4\right) \log r+C$ for large $r$; this yields that $\beta<+\infty$, a contradiction. Now by the fact that $f e^{v}<1$ for the entire solutions, there are only two possibilities that either (i) $r^{2 N_{1}} e^{v(r)}$ increases for all $r>0$, or (ii) $r^{2 N_{1}} e^{v(r)}$ decreases in an interval $\left(r_{0},+\infty\right)$ for some $r_{0}>0$. In the case (i), we have $\beta=2 N_{1}$ and $f(r) e^{v(r)} \rightarrow 1$ as $r \rightarrow+\infty$ where we note that if $f(r) e^{v(r)} \rightarrow c<1$, it will contradict (19). In the case (ii), from Lemma 2.2 we have $f(r) e^{v(r)} \rightarrow 0$ as $r \rightarrow+\infty$, which indicates that $\beta>2 N_{1}+2$ by (19). By the way, in either case of the entire solution, we have

$$
v(r)=-\beta \log r+O(1), \quad r \rightarrow+\infty .
$$

The classification (15) is concluded.

Taking account of the initial value $s$ for the solution $v(r)=v(r ; s)$ of (14) depending on $s$, we set

$$
E(r, s)=\left[r v^{\prime}(r ; s)\right]^{2}-r^{2} f^{2}(r) e^{2 v(r ; s)}+2 r^{2} f(r) e^{v(r ; s)} .
$$

Multiplying (14) by the factor $r v^{\prime}$ and taking integration both sides, we obtain the following variational identity:

$$
\frac{1}{2} E(r, s)=\int_{0}^{r} t g(t) f(t) e^{v(t ; s)}\left[1-f(t) e^{v(t ; s)}\right] d t+\int_{0}^{r} t f^{2}(t) e^{2 v(t ; s)} d t,
$$


where $g(t)=\left[t f^{\prime}(t) / f(t)\right]+2$. We define

$J_{i}=\{s \in \mathbb{R}: v(r ; s)$ is of the $i$-Type according to (15) $\}, \quad i=0,1, \infty$.

Clearly, $\mathbb{R}=J_{0} \cup J_{1} \cup J_{\infty} . E(r, s)>0$ near $r=0$ for any $s \in \mathbb{R}$. We remark that $s \in J_{\infty}$ if and only if $E\left(r_{0}, s\right)=0$ for some $r_{0}>0$. In fact, if $s^{*} \in J_{0} \cup J_{1}$, then

$$
\frac{\partial E}{\partial r}\left(r, s^{*}\right)=r g(r) f(r) e^{v\left(r ; s^{*}\right)}\left[1-f(r) e^{v\left(r ; s^{*}\right)}\right]+r f^{2}(r) e^{2 v\left(r ; s^{*}\right)}
$$

is positive for all $r>0$, where we apply the fact that $f e^{v}<1$. Thus $E\left(r, s^{*}\right)$ is positive whenever $r>0$. On the other hand, letting $s_{*} \in J_{\infty}$, it is not hard to see that $E\left(r, s_{*}\right) \rightarrow-\infty$ as $r \rightarrow \omega$ in the maximal existence interval $I_{\max }=[0, \omega)$ of the solution $v\left(r ; s_{*}\right)$. Hence $E\left(r, s_{*}\right)$ must vanish at some site $r=r_{0}>0$. In addition, we conclude the following lemma.

Lemma 2.3. Both $J_{0}$ and $J_{\infty}$ are open sets. Moreover, $J_{0} \cup J_{1}$ is bounded from above.

Proof. The assertion of the lemma about $J_{0}$ can be concluded readily from Lemma 2.2 by means of the continuous dependence of $v(r ; s)$ on the parameter $s$. We omit the details and straightforwardly account for the set $J_{\infty}$. To show that $J_{\infty}$ is open, we take advantage of the variational identity (21). Let $s_{0} \in J_{\infty}$. Then there exists $r_{0}>0$ such that $E\left(r_{0}, s_{0}\right)=0$. By definition, we have

$$
\left[r_{0} v^{\prime}\left(r_{0}\right)\right]^{2}+r_{0}^{2} f\left(r_{0}\right) e^{v\left(r_{0}\right)}\left[2-f\left(r_{0}\right) e^{v\left(r_{0}\right)}\right]=E\left(r_{0}, s_{0}\right)=0, \quad v(r)=v\left(r ; s_{0}\right),
$$

implying that $f\left(r_{0}\right) e^{v\left(r_{0}\right)}>2$. Hence by a rearrangement in the formula (22),

$$
\frac{\partial E}{\partial r}\left(r, s_{0}\right)=2 r_{0}^{2} f^{\prime}\left(r_{0}\right) e^{v\left(r_{0}\right)}\left[1-f\left(r_{0}\right) e^{v\left(r_{0}\right)}\right]+2 r_{0} f\left(r_{0}\right) e^{v\left(r_{0}\right)}\left[2-f\left(r_{0}\right) e^{v\left(r_{0}\right)}\right]<0 .
$$

Using the Implicit Function Theorem, $r$ can be represented as a $C^{1}$-function of $s$ in a neighborhood $I_{\epsilon}\left(s_{0}\right)=$ $\left(s_{0}-\epsilon, s_{0}+\epsilon\right)$ of $s_{0}$, with a small $\epsilon>0$, such that $r\left(s_{0}\right)=r_{0}$ and

$$
E(r(s), s)=0, \quad s \in I_{\epsilon}\left(s_{0}\right) .
$$

Therefore $I_{\epsilon}\left(s_{0}\right) \subset J_{\infty}$. As for the remaining part of the lemma, we first show that the set $J_{0}$ has an upper bound. Assume contrarily that there exists a sequence of real numbers $s_{j} \in J_{0}$ such that $s_{j} \rightarrow+\infty$ as $j \rightarrow+\infty$. Through an adaptation of the device in [4], we let

$$
w_{j}(r)=v\left(e^{-s_{j} /\left(2 N_{K}+1\right)} r ; s_{j}\right)-s_{j} .
$$

Assume $N_{1}>N_{2}>\cdots>N_{K}$ (without loss of generality). Then, by a direct computation from (14), $w_{j}$ solves the equation

$$
\left\{\begin{array}{l}
w_{j}^{\prime \prime}(r)+\frac{1}{r} w_{j}^{\prime}(r)=G_{j}^{2}(r) e^{2 w_{j}(r)}-e^{-s_{j} /\left(2 N_{k}+1\right)} G_{j}(r) e^{w_{j}(r)}, \quad r>0, \\
w_{j}(0)=0, \quad w_{j}^{\prime}(0)=0,
\end{array}\right.
$$

where

$$
G_{j}(r)=\sum_{m=1}^{K} e^{-2 s_{j}\left(N_{m}-N_{K}\right)} r^{2 N_{m}} .
$$

Note that $v\left(r ; s_{j}\right)$ is decreasing in $r ; w_{j}$ is thus decreasing and the right hand side of Eq. (23) converges uniformly on any compact interval. By passing to a subsequence, it follows that $w_{j}$ approaches to a function $\tilde{w}$, which solves

$$
\left\{\begin{array}{l}
\tilde{w}^{\prime \prime}(r)+\frac{1}{r} \tilde{w}^{\prime}(r)=r^{4 N_{K}} e^{2 \tilde{w}(r)}, \quad r>0 \\
\tilde{w}(0)=0, \quad \tilde{w}^{\prime}(0)=0
\end{array}\right.
$$

This is impossible because any solution of (24) is increasing and blows up at finite $r$. So that $J_{0}$ is bounded from above. The argument for $s_{j} \in J_{1}$ is the same. We omit it here. 
To make out how the solution $v$ depends on $s$, we consider the function $\varphi(r)=\varphi(r ; s)=(\partial v / \partial s)(r ; s)$. Directly taking derivative on Eq. (14), $\varphi$ solves the following equation

$$
\left\{\begin{array}{l}
\varphi^{\prime \prime}(r)+\frac{1}{r} \varphi^{\prime}(r)=f(r) e^{v(r)}\left[2 f(r) e^{v(r)}-1\right] \varphi(r), \quad v(r)=v(r ; s), \\
\varphi(0)=1, \quad \varphi^{\prime}(0)=0 .
\end{array}\right.
$$

Lemma 2.4. The functions $\varphi(r ; s)$ and $(\partial \varphi / \partial r)(r ; s)$ are continuously dependent on sor any fixed $r$.

Proof. Let $s^{\prime} \in \mathbb{R}$. Consider the function $w(r)=w(r ; s)=\varphi(r ; s)-\varphi\left(r ; s^{\prime}\right)$ for $\left|s-s^{\prime}\right| \leq \delta$ with a small $\delta>0$. To conclude the lemma, it suffices to show that $w(r ; s) \rightarrow 0$ as $s \rightarrow s^{\prime}$ for any fixed $r$. In fact, by a direct computation from (25),

$$
w^{\prime \prime}(r)+\frac{1}{r} w^{\prime}(r)=P(r ; s) w(r)+Q(r ; s),
$$

where $P, Q$ are functions of $r$, depending on $s$ and given by

$$
\begin{aligned}
& P(r ; s)=f(r) e^{v(r ; s)}\left[2 f(r) e^{v(r ; s)}-1\right], \\
& Q(r ; s)=f(r)\left\{2 f(r)\left[e^{v(r ; s)}+e^{v\left(r ; s^{\prime}\right)}\right]-1\right\}\left[e^{v(r ; s)}-e^{v\left(r ; s^{\prime}\right)}\right] \varphi\left(r ; s^{\prime}\right) .
\end{aligned}
$$

So that $w$ satisfies the integral equation

$$
w(r)=\int_{0}^{r} t\left(\log \frac{r}{t}\right) P(t ; s) w(t) d t+\int_{0}^{r} t\left(\log \frac{r}{t}\right) Q(t ; s) d t .
$$

Therefore, by the continuity of $v(r ; s)$, we have

$$
\begin{aligned}
|w(r ; s)| & \leq\left(\int_{0}^{r} t|Q(t ; s)| \log \frac{r}{t} d t\right) \exp \left(\int_{0}^{r} t|P(t ; s)| \log \frac{r}{t} d t\right) \\
& \leq C \int_{0}^{r}|Q(t ; s)| d t \\
& \leq \widetilde{C} \sup _{t \in[0, r]}\left|v(t ; s)-v\left(t ; s^{\prime}\right)\right| \rightarrow 0
\end{aligned}
$$

as $s \rightarrow s^{\prime}$. This concludes the proof.

Lemma 2.5. If $s_{0} \in J_{0}$, then there exist numbers $C, R, \epsilon>0$ such that

$$
|\varphi(r ; s)| \leq C \log r
$$

for all $r \geq R$ and $s \in J_{0} \cap\left\{s \in \mathbb{R}:\left|s-s_{0}\right|<\epsilon\right\}$.

Proof. Let $s_{0} \in J_{0}$. We show that $\varphi_{0}(r)=\varphi\left(r ; s_{0}\right)$ does not change sign for $r$ sufficiently large. In fact, by comparison $\varphi$ with the function $w_{c}(r)=r v^{\prime}(r)+c$, here $v(r)=v\left(r ; s_{0}\right)$ and $c$ being a constant, we have

$$
\begin{aligned}
{\left[r\left(\varphi_{0} w_{c}^{\prime}-w_{c} \varphi_{0}^{\prime}\right)\right]_{r_{1}}^{r_{2}} } & =\int_{r_{1}}^{r_{2}} \varphi_{0}\left(r w_{c}^{\prime}\right)^{\prime}-w_{c}\left(r \varphi_{0}^{\prime}\right)^{\prime} d r \\
& =\int_{r_{1}}^{r_{2}} r\left[2 f e^{v}\left(f e^{v}-1\right)+g_{c} f e^{v}\left(2 f e^{v}-1\right)\right] \varphi_{0} d r
\end{aligned}
$$


where $g_{c}(r)=\left[\left(r f^{\prime}(r)\right) / f(r)\right]-c$. Since $r v^{\prime}(r)$ vanishes at $r=0$ and decreases to $-\beta$ with $\beta=\beta\left(s_{0}\right)>2 N_{1}+2$ for the 0-Type solution, there can be $\lambda \in\left(2 N_{1}+2, \beta\right)$ such that $w_{\lambda}\left(r_{\lambda}\right)=0$ for some $r_{\lambda}>0$, whereas $w_{\lambda}>0$ in the interval $\left(0, r_{\lambda}\right)$ and $w_{\lambda}<0$ in $\left(r_{\lambda},+\infty\right)$. If $\varphi_{0}$ changes sign no matter how large $r$ is, it is possible to select two successive zeros $r_{1}, r_{2}>r_{\lambda}$ of $\varphi_{0}$ such that $r_{1}<r_{2}, \varphi_{0}^{\prime}\left(r_{1}\right)>0, \varphi_{0}^{\prime}\left(r_{2}\right)<0$ and $\varphi_{0}>0$ in between. Note that $g_{\lambda}<-2$ and from (20) we have $f e^{v} \approx r^{-2-\sigma}$ near infinity, $\sigma>0$. Hence the left hand side of (27) is positive, but the right hand side is negative. This is a contradiction. Now we consider the case $\varphi_{0}>0$ in $\left(r_{0},+\infty\right)$ for some large $r_{0}$; the argument for its negative counterpart is similar. From the expression

$$
r \varphi_{0}^{\prime}(r)=r_{0} \varphi_{0}^{\prime}\left(r_{0}\right)+\int_{r_{0}}^{r} t f(t) e^{v(t)}\left[2 f(t) e^{v(t)}-1\right] \varphi_{0}(t) d t,
$$

we have $r_{0} \varphi_{0}^{\prime}\left(r_{0}\right)-C r^{-\sigma} \leq r \varphi_{0}^{\prime}(r) \leq r_{0} \varphi_{0}^{\prime}\left(r_{0}\right)$ for $r>r_{0}$ and some positive constant $C$, where we use the fact that $f e^{v} \ll 1$ in $\left(r_{0},+\infty\right)$ for $r_{0}$ sufficiently large. Thus, there is $C_{1}>0$ such that

$$
\left|\varphi_{0}(r)\right| \leq C_{1} \log r, \quad r \geq r_{0} .
$$

To conclude the lemma, we need to show that there exist $\sigma, c_{0}>0$ such that

$$
f(r) e^{v(r ; s)} \leq c_{0} r^{-2-\sigma}
$$

for any $s$ in a neighborhood of $s_{0}$ and $r$ sufficiently large. In fact, taking $\sigma>0$ so small that

$$
\beta=\beta\left(s_{0}\right):=\int_{0}^{\infty} r f(r) e^{v\left(r ; s_{0}\right)}\left[1-f(r) e^{v\left(r ; s_{0}\right)}\right] d r>2\left(N_{1}+1\right)+3 \sigma,
$$

we have

$$
\begin{aligned}
r v^{\prime}\left(r ; s_{0}\right) & =-\beta+\int_{r}^{\infty} t f(t) e^{v\left(t ; s_{0}\right)}\left[1-f(t) e^{v\left(t ; s_{0}\right)}\right] d t \\
& <-2\left(N_{1}+1\right)-3 \sigma+c_{1} r^{2 N_{1}+2-\beta} \\
& <-2\left(N_{1}+1\right)-2 \sigma, \quad r \geq r_{1}
\end{aligned}
$$

for some $r_{1} \geq r_{0}$. Assume $J_{0} \supset\left(s_{0}-\epsilon_{0}, s_{0}+\epsilon_{0}\right)$ for some $\epsilon_{0}>0$. By the continuity of $v^{\prime}(r ; \cdot)$ as a function of $s$, it is possible to extract a small $\epsilon_{1} \in\left(0, \epsilon_{0}\right)$ such that

$$
r v^{\prime}(r ; s) \leq r v^{\prime}\left(r_{1} ; s\right)<r v^{\prime}\left(r_{1} ; s_{0}\right)+\sigma<-2\left(N_{1}+1\right)-\sigma,
$$

and hence $v(r ; s) \leq c_{2}-\left(2 N_{1}+2+\sigma\right) \log r$ for all $\left|s-s_{0}\right|<\epsilon_{1}$ and $r \geq r_{1}$. This concludes the proof.

\section{Theorem 2.2. The function}

$$
\beta(s)=\int_{0}^{\infty} r f(r) e^{v(r ; s)}\left[1-f(r) e^{v(r ; s)}\right] d r
$$

is differentiable on $J_{0}$ and $\sup _{s \in J_{0}} \beta(s)=+\infty$.

Proof. The differentiability of $\beta(s)$ follows readily from Lemma 2.4 by a direct computation. To see the unboundedness of $\beta$, we pick $s_{0} \in J_{0}$ and define $s^{*}=\sup \left\{s^{\prime}:\left[s_{0}, s^{\prime}\right) \subset J_{0}\right\}$. By Lemma 2.3, $s^{*} \in J_{1}$. We show that $\beta(s)$ goes to infinity as $s \rightarrow s^{*}$. As a matter of fact, if we consider the sequence $v_{j}(r)=v\left(r ; s_{j}\right)$ with $s_{j} \in\left(s_{0}, s^{*}\right)$ and $s_{j} \rightarrow s^{*}$ as $j \rightarrow+\infty$, from the variational identity (21), we have 


$$
\begin{aligned}
\frac{1}{2} \beta^{2}\left(s_{j}\right) & =\int_{0}^{\infty} t g(t) f(t) e^{v_{j}(t)}\left[1-f(t) e^{v_{j}(t)}\right] d t+\int_{0}^{\infty} t f^{2}(t) e^{2 v_{j}(t)} d t \\
& \geq \int_{0}^{\infty} t f^{2}(t) e^{2 v_{j}(t)} d t .
\end{aligned}
$$

Assume $\beta\left(s_{j}\right)$ is bounded. Then $r f^{2}(r) e^{2 v\left(r ; s^{*}\right)} \in L^{1}(\mathbb{R})$. This contradicts the fact that $f(r) e^{v\left(r ; s^{*}\right)} \rightarrow 1$ as $r \rightarrow+\infty$. So that $\beta(s)$ is not bounded.

Lemma 2.6. Assume $N_{1}-N_{K} \leq 1$. If $s^{*} \in J_{1}$, then there exists $\mu>0$ such that

$$
\varphi\left(r ; s^{*}\right) \geq \mu
$$

for all $r>0$. Moreover, $\lim _{r \rightarrow+\infty} \varphi\left(r ; s^{*}\right) \rightarrow+\infty$.

Proof. Note first that, from Theorem 2.1, the function $r v^{\prime}\left(r ; s^{*}\right)$ is strictly greater than $-2 N_{1}$ for all $r>0$ and approaches to $-2 N_{1}$ as $r \rightarrow+\infty$. So that the function

$$
w(r)=r v^{\prime}(r)+2 N_{1}, \quad v(r)=v\left(r ; s^{*}\right),
$$

is positive all the time and $w(r) \rightarrow 0$ as $r \rightarrow+\infty$. Applying the identity (27) between the functions $w(r)$ and $\varphi(r)=$ $\varphi\left(r ; s^{*}\right)$ with the substitution $r_{1}=0, r_{2}=R$ and $c=2 N_{1}$, gives

$$
\begin{aligned}
R\left[\varphi(R) w^{\prime}(R)-w(R) \varphi^{\prime}(R)\right] & =\int_{0}^{R} \varphi\left(r w^{\prime}\right)^{\prime}-w\left(r \varphi^{\prime}\right)^{\prime} d r \\
& =\int_{0}^{R} r\left[\left(g_{c}+2\right) f e^{v}\left(f e^{v}-1\right)+g_{c} f^{2} e^{2 v}\right] \varphi d r<0
\end{aligned}
$$

for all $R>0$, where we make use of the fact that $-2 \leq g_{c} \leq 0$ for $c=2 N_{1}$. Applying (28), we show that $\varphi>0$ as follows. In fact, assuming contrarily that $\varphi$ has the first zero at $r=\sigma>0$ and letting $R=\sigma$, it follows that the left hand side (LHS) of the identity (28) is positive while the right hand side (RHS) is negative. This contradiction indicates that $\varphi(r)$ must be positive for all $r>0$. To conclude the proof, it suffices to show that $\varphi$ is not a bounded function. Assume contrarily that $\varphi$ is bounded. Then from (25) it is not hard to see that

$$
\lim _{r \rightarrow+\infty} r \varphi^{\prime}(r)=0 .
$$

Now applying (28) again and letting $R \rightarrow+\infty$, the LHS of (28) approaches to 0 , while the RHS approaches to a negative number; that is a contradiction. Hence the function $\varphi$ is unbounded and thus from (25) we note, in addition, that $\varphi$ cannot oscillate near infinity. So that $\varphi(r) \rightarrow+\infty$ as $r \rightarrow+\infty$. Therefore, $\varphi$ has a positive lower bound.

Remark 2.1. For $s^{*} \in J_{1}$, from Lemma 2.5 and (25) it follows that

$$
\begin{aligned}
\varphi(r) & =\varphi\left(r_{0}\right)+\int_{r_{0}}^{r} t\left(\log \frac{r}{t}\right) f(t) e^{v(t)}\left[2 f(t) e^{v(t)}-1\right] \varphi(t) d t \\
& \geq \varphi\left(r_{0}\right)+\frac{1}{2} \int_{r_{0}}^{r} t\left(\log \frac{r}{t}\right) \varphi(t) d t, \quad r \geq r_{0},
\end{aligned}
$$

for $r_{0}>0$ sufficiently large, where $v(r)=v\left(r ; s^{*}\right)$ and $\varphi(r)=\varphi\left(r ; s^{*}\right)$. Thus, we may conclude further that $\varphi\left(r ; s^{*}\right)$ grows exponentially near infinity; in fact, the inequality

$$
\varphi\left(r ; s^{*}\right) \geq c_{1} \exp \left(c_{2} r^{2}\right)
$$

holds true in a neighborhood of $+\infty$ for which $c_{1}, c_{2}>0$ are suitable constants. 
Proof of Theorem 1.2. From Lemma 2.3, it is clear that the set $J_{1}$ is nonempty. To conclude the theorem, we are going to show that $J_{1}$ consists of a single point. Let $s^{*} \in J_{1}$. In view of Lemmas 2.4 and 2.6, as well as the continuity of $v(r ; s)$, it is possible to select $r_{0}>0$ and $\delta>0$ such that

(a) $2 f(r) e^{v\left(r, s^{*}\right)}>1$ for $r \leq r_{0}$;

(b) $2 f\left(r_{0}\right) e^{v\left(r_{0}, s\right)}>1$ and $\varphi^{\prime}\left(r_{0} ; s\right)>0$ for $s \in I_{\delta}=\left(s^{*}-\delta, s^{*}+\delta\right)$;

(c) $\varphi(r ; s) \geq \lambda>0$ for $(r, s) \in\left[0, r_{0}\right] \times I_{\delta}$.

We set $I_{\delta}^{+}=\left(s^{*}, s^{*}+\delta\right)$ and $I_{\delta}^{-}=\left(s^{*}-\delta, s^{*}\right)$. Letting $\left[0, \omega_{s}\right)$ with $\omega_{s} \in \mathbb{R} \cup\{+\infty\}$ be the maximal existence interval for the solution $v(\cdot)=v(\cdot ; s)$ that solves $(14)$, we have the following conclusion:

Claim 1. $\varphi(r, s) \geq \lambda$ and $2 f(r) e^{v(r, s)}>1$ for all $s \in I_{\delta}^{+}$and $r \in\left[r_{0}, \omega_{s}\right)$.

Assume our assertion fails. Then by the statements (b) and (c), we may extract $s^{\prime} \in I_{\delta}^{+}$and $r_{1} \in\left(r_{0}, \omega_{s^{\prime}}\right)$ such that $\varphi^{\prime}\left(r ; s^{\prime}\right)>0$ for $r \in\left[r_{0}, r_{1}\right)$ and $\varphi^{\prime}\left(r_{1} ; s^{\prime}\right)=0$. Thus, from the statement (a), we have $2 f(r) e^{v\left(r, s^{\prime}\right)}>1$ for $r \in\left[r_{0}, r_{1}\right)$. So that

$$
0=r_{1} \varphi^{\prime}\left(r_{1} ; s^{\prime}\right)=r_{0} \varphi^{\prime}\left(r_{0} ; s^{\prime}\right)+\int_{r_{0}}^{r_{1}} f(t) e^{v\left(t ; s^{\prime}\right)}\left[2 f(t) e^{v\left(t ; s^{\prime}\right)}-1\right] \varphi\left(t ; s^{\prime}\right) d t>0,
$$

which is a contradiction. So we conclude Claim 1. Since $f(r) e^{v\left(r ; s_{0}\right)} \rightarrow 0$ as $r \rightarrow+\infty$ for any $s \in J_{0}$, it follows from Claim 1 that $I_{\delta}^{+} \cap J_{0}$ is empty. Now we continue to the following claims.

Claim 2. If there exist two points $s_{1}, s_{2} \in J_{1} \cap I_{\delta}$, then there must be a point $s^{\prime} \in J_{\infty}$ which lies between $s_{1}$ and $s_{2}$.

To see this, we assume, in contrast to the assertion, that $v(r ; s)$ is an entire solution of (14) for all $s \in\left(s_{1}, s_{2}\right)$ where we suppose $s_{2}>s_{1}$. This implies that $\left(s_{1}, s_{2}\right) \cap J_{0}$ is empty. Indeed, if there is $s_{0} \in\left(s_{1}, s_{2}\right) \cap J_{0}$, then, since $f(r) e^{v\left(r ; s_{0}\right)} \rightarrow 0$ as $r \rightarrow+\infty$, we can find $\left(r^{\prime}, s^{\prime}\right) \in\left[r_{0},+\infty\right) \times I_{\delta}$ such that $\varphi^{\prime}\left(r^{\prime}, s^{\prime}\right)=0$. Consequently, as have done with (29), we may arrive at a contradiction. Now we conclude that $\left(s_{1}, s_{2}\right) \subset J_{1}$. Therefore, we have

$$
v\left(r ; s_{2}\right)-v\left(r ; s_{1}\right)=\varphi(r ; \bar{s})\left(s_{2}-s_{1}\right) \geq \lambda\left(s_{2}-s_{1}\right) \equiv c>0, \quad \bar{s} \in\left(s_{1}, s_{2}\right),
$$

for all $r \geq r_{0}$, where we apply the statement (c) and the formula (29) again. However, this contradicts the fact that both $f(r) e^{v\left(r ; s_{1}\right)}$ and $f(r) e^{v\left(r ; s_{2}\right)}$ approach to 1 as $r \rightarrow+\infty$.

Claim 3. If $s_{\infty} \in I_{\delta} \cap J_{\infty}$, then $\left[s_{\infty}, s^{*}+\delta\right) \subset J_{\infty}$.

The proof of Claim 3 is essentially the same as that of Claim 1. We omit it here. From Claims 1, 2 and 3, we conclude that $I_{\delta}^{+} \subset J_{\infty}$ and $I_{\delta}^{-} \subset J_{0}$. If $J_{1}$ consists of more than one elements, then, in terms of Lemma 2.3, it is possible to select $s_{1}, s_{2} \in J_{1}$ with $s_{1}<s_{2}$ such that either the interval $\left(s_{1}, s_{2}\right) \subset J_{0}$ or $\left(s_{1}, s_{2}\right) \subset J_{\infty}$. In either case, it contradicts the conclusion we have just mentioned. Therefore, the proof is complete.

\section{Sharp lower bound of flux for $N_{1} \leq 2 N_{K}+1$}

In the preceding section we have proved that it is possible to find a radially symmetric nontopological solutions of the system (2) whose flux assumes an arbitrarily large value. We are going to make an effort to grasp the optimal lower bound for the value $\beta$ associated with the (radial) nontopological solutions. Notice that, letting $r \rightarrow+\infty$ in the identity (21), we have $\beta>4 N_{K}+4$. Combining this with the constraint (19) thus gives

$$
\beta>\max \left\{2 N_{1}+2,4 N_{K}+4\right\} .
$$

To sharpen the lower bound, we take advantage of the radially symmetric solutions of the Liouville system in correspondence with (2), namely 


$$
\Delta v_{j}=-\sum_{k=1}^{K} e^{v_{k}}+4 \pi N_{j} \delta_{0} \quad \text { in } \mathbb{R}^{2}, j=1, \ldots, K,
$$

and take account of the value

$$
\omega=\frac{1}{2 \pi} \int_{\mathbb{R}^{2}}\left(\sum_{k=1}^{K} e^{v_{k}}\right) d \mathbf{x}
$$

Letting $v_{j}(r)=\hat{v}(r)+\log c_{j}+2 N_{j} \log r$ for $c_{j}>0, j=1, \ldots, K$, we reduce (31) to

$$
\left\{\begin{array}{l}
\hat{v}^{\prime \prime}+\frac{1}{r} \hat{v}^{\prime}=-f e^{\hat{v}}, \quad r>0, \\
\hat{v}(0 ; s)=s \in \mathbb{R}, \quad \omega(s)=\int_{0}^{\infty} r f(r) e^{\hat{v}(r ; s)} d r,
\end{array}\right.
$$

where $f(r)=\sum_{j=1}^{K} c_{j} r^{2 N_{j}}$. It is clearly to see that $\omega(s)$ is continuous on $(-\infty, \infty)$ and $\hat{v}(r ; s)=-\omega(s) \log r+O(1)$ as $r \rightarrow+\infty$.

Remark 3.1. An important problem is concerned with the structure of total curvature $\omega(s)$ for generalized Gaussian curvature equation that has been extensively studied by many authors. From Cheng and Lin [6] and (32), we see that

i) if $N_{1} \leq 2 N_{K}+1$, then $\lim _{s \rightarrow-\infty} \omega(s)=4\left(N_{1}+1\right)$ and $\lim _{s \rightarrow+\infty} \omega(s)=4\left(N_{K}+1\right)$;

ii) if $N_{1}>2 N_{K}+1$, then $\lim _{s \rightarrow-\infty} \omega(s)=4\left(N_{1}+1\right)$ and $\lim _{s \rightarrow+\infty} \omega(s)=4\left(N_{1}-N_{K}\right)$.

This implies that $\omega(s)$ is a bounded function. To attain a greatest lower bound of $\omega(s)$, we take account of the variational identity

$$
\frac{1}{2}\left[r \hat{v}^{\prime}(r)\right]^{2}-r^{2} f^{\prime}(r) e^{\hat{v}(r)}-2 r f(r) e^{\hat{v}(r)}=\int_{0}^{r} t\left[\frac{t f^{\prime}(t)}{f(t)}+2\right] f(t) e^{\hat{v}(t)} d t,
$$

which is obtained by multiplying Eq. (32) by the factor $r v^{\prime}$ and using integration by part on both sides. Adding $\left(4 N_{K}+4\right) r \hat{v}^{\prime}(r)$ to the identity and letting $r \rightarrow+\infty$, it follows that

$$
\omega\left(\omega-4 N_{K}-4\right)=2 \int_{0}^{\infty} t\left[\frac{t f^{\prime}(t)}{f(t)}-2 N_{K}\right] f(t) e^{\hat{v}(t)} d t>0 .
$$

Hence it is necessary that $\omega>4 N_{K}+4$ in order for (32) to be solvable. This, together with the statement i) above, indicates that the lower bound $4 N_{K}+4$ for $\omega$ is optimal provided $N_{1} \leq 2 N_{K}+1$. When $N_{1}>2 N_{K}+1$, it is not clear whether Eq. (32) has any solution with total curvature between $2\left(N_{1}+1\right)$ and $4\left(N_{1}-N_{K}\right)$. It could be natural to conjecture that $\omega(s)$ is not a monotone function in case the solution behaves too dramatically. However, as shown in [9], the function $\partial \hat{v}(r ; s) / \partial s$ changes sign at least twice; it seems to be a choke point for the monotonicity of $\omega$ to be established.

In fact, $\omega(s)$ is not a monotone function on $(-\infty, \infty)$ when $N_{1}>2 N_{K}+1$ and we will prove it in the next section. Here is an existence result that depends on the system (31).

Theorem 3.1. Let $v_{1}, \ldots, v_{K}$ solve (31) with $\omega$ being given. Then there exists a family of the nontopological solutions $\left(u_{1}^{\varepsilon}, \ldots, u_{K}^{\varepsilon}\right)$ of the system (2) such that $\beta_{\varepsilon}$ therein is dependent continuously on $\varepsilon$ in a small neighborhood of the origin and

$$
\beta_{\varepsilon} \rightarrow \omega, \quad \text { as } \varepsilon \rightarrow 0 .
$$


In advance of going into the details of the proof of Theorem 3.1, we now carry out the conclusion of Theorem 1.1 for the case: $N_{1} \leq 2 N_{K}+1$ beforehand.

Proof of Theorem 1.1. That $\beta \in\left\{2 N_{1}\right\} \cup\left(\omega_{1},+\infty\right)$ with $\omega_{1}=\max \left\{2 N_{1}+2,4 N_{K}+4\right\}$ as well as the first assertion of Theorem 1.1 follows readily from Theorems 2.1, 2.2 and (30). To complete the proof, we consider Eq. (32). From Remark 3.1, Eq. (32) is solvable for any prescribed value $\omega \in\left(\omega_{*}, \omega^{*}\right)$ where $\omega^{*}=4 N_{1}+4$ and $\omega_{*}=4 N_{K}+4$ by $N_{1} \leq 2 N_{K}+1$. So that Theorem 3.1 together with Theorem 2.2 indicates the solvability of (13) for any prescribed $\beta \in\left(\omega_{*},+\infty\right)$; this concludes the proof of (b). Finally, the result (c) can be obtained by Theorem 4.1 and hence we complete this proof of Theorem 1.1.

On purpose to prove Theorem 3.1, we make some preliminary settings as follows. Define the inner products $\langle,\rangle_{X_{\alpha}}$ and $\langle,\rangle_{Y_{\alpha}}, 0<\alpha<1$, for functions in the spaces $L_{\text {loc }}^{2}\left(\mathbb{R}^{2}\right)$ and $W_{\text {loc }}^{2,2}\left(\mathbb{R}^{2}\right)$ respectively by

$$
\begin{aligned}
& \langle u, v\rangle_{X_{\alpha}}=\int_{\mathbb{R}^{2}}\left(1+|\mathbf{x}|^{2+\alpha}\right) u v d \mathbf{x}, \quad u, v \in L_{\mathrm{loc}}^{2}\left(\mathbb{R}^{2}\right), \\
& \langle u, v\rangle_{Y_{\alpha}}=\langle\Delta u, \Delta v\rangle_{X_{\alpha}}+\int_{\mathbb{R}^{2}} \frac{u v}{1+|\mathbf{x}|^{2+\alpha}} d \mathbf{x}, \quad u, v \in W_{\mathrm{loc}}^{2,2}\left(\mathbb{R}^{2}\right) ;
\end{aligned}
$$

we define two specific function spaces $X_{\alpha}$ and $Y_{\alpha}$ in the following:

$$
\begin{aligned}
& X_{\alpha}=\left\{u \in L_{\mathrm{loc}}^{2}\left(\mathbb{R}^{2}\right):\langle u, u\rangle_{X_{\alpha}}<+\infty\right\}, \\
& Y_{\alpha}=\left\{u \in W_{\mathrm{loc}}^{2,2}\left(\mathbb{R}^{2}\right):\langle u, u\rangle_{Y_{\alpha}}<+\infty\right\} .
\end{aligned}
$$

Clearly, $X_{\alpha}$ and $Y_{\alpha}$ are Hilbert spaces with the inner products $\langle,\rangle_{X_{\alpha}}$ and $\langle,\rangle_{Y_{\alpha}}$ while the $X_{\alpha}$-norm and $Y_{\alpha}$-norm are given respectively by

$$
\|u\|_{X_{\alpha}}=\sqrt{\langle u, u\rangle_{X_{\alpha}}}, \quad\|u\|_{Y_{\alpha}}=\sqrt{\langle u, u\rangle_{Y_{\alpha}}} .
$$

We recall the imbeddings $X_{\alpha} \hookrightarrow L^{1}\left(\mathbb{R}^{2}\right)$ and $Y_{\alpha} \hookrightarrow C_{\mathrm{loc}}^{0}\left(\mathbb{R}^{2}\right)$ which are introduced in [3]. Moreover, the following properties of $Y_{\alpha}$ [3, Lemma 1.1] are useful in the construction of solutions.

Proposition 3.1. There exists a constant $C>0$ such that for all $u \in Y_{\alpha}$,

$$
|u(\mathbf{x})| \leq C\|u\|_{Y_{\alpha}}\left(\log ^{+}|\mathbf{x}|+1\right), \quad \mathbf{x} \in \mathbb{R}^{2},
$$

where $\log ^{+}|\mathbf{x}|=\max \{0, \log |\mathbf{x}|\}$.

Let $X_{\alpha}^{r}$ and $Y_{\alpha}^{r}$ be the spaces consisting of the radial functions in $X_{\alpha}$ and $Y_{\alpha}$ defined in (34) respectively. We equip the spaces

$$
\prod_{K} X_{\alpha}^{r}=\underbrace{X_{\alpha}^{r} \times \cdots \times X_{\alpha}^{r}}_{K} \text { and } \prod_{K} Y_{\alpha}^{r}=\underbrace{Y_{\alpha}^{r} \times \cdots \times Y_{\alpha}^{r}}_{K}
$$

with the inner products

$$
\left\langle u^{(1)}, u^{(2)}\right\rangle_{X_{\alpha}^{K}}=\sum_{j=1}^{K}\left\langle u_{j}^{(1)}, u_{j}^{(2)}\right\rangle_{X_{\alpha}}, \quad\left\langle v^{(1)}, v^{(2)}\right\rangle_{Y_{\alpha}^{K}}=\sum_{j=1}^{K}\left\langle v_{j}^{(1)}, v_{j}^{(2)}\right\rangle_{Y_{\alpha}},
$$

where $u^{(i)}=\left(u_{1}^{(i)}, \ldots, u_{K}^{(i)}\right), v^{(i)}=\left(v_{1}^{(i)}, \ldots, v_{K}^{(i)}\right)$ for $u_{j}^{(i)} \in X_{\alpha}^{r}, v_{j}^{(i)} \in Y_{\alpha}^{r}, i=1,2$ and $j=1, \ldots, K$.

In order to use a solution of (31) as source material to manufacture a nontopological solution for the system (2), we define auxiliary functions as follows:

$$
\eta_{j}(r)=\frac{1}{\varepsilon^{2}} u_{j}\left(\frac{r}{\varepsilon}\right)-\frac{1}{\varepsilon^{2}} v_{j}(r)-w(r)-\frac{2}{\varepsilon^{2}} \log \varepsilon, \quad j=1, \ldots, K,
$$


where $w_{j}$ is independent of $\varepsilon$ solving

$$
\Delta w+\left(\sum_{k=1}^{K} e^{v_{k}}\right) w=\left(\sum_{k=1}^{K} e^{v_{k}}\right)^{2}
$$

It is not hard to see that

$$
|w(r)| \leq c \log (1+r), \quad r>0,
$$

for some $c>0$. Now set

$$
\begin{aligned}
P_{j}\left(\varepsilon, \eta_{1}, \ldots, \eta_{K}\right)= & \Delta \eta_{j}-\left(\sum_{k=1}^{K} e^{v_{k}+\varepsilon^{2}\left(\eta_{k}+w\right)}\right)^{2}+\varepsilon^{-2} \sum_{k=1}^{K} e^{v_{k}+\varepsilon^{2}\left(\eta_{k}+w\right)} \\
& -\varepsilon^{-2} \sum_{k=1}^{K} e^{v_{k}}-\left(\sum_{k=1}^{K} e^{v_{k}}\right) w+\left(\sum_{k=1}^{K} e^{v_{k}}\right)^{2}, \quad j=1, \ldots, K .
\end{aligned}
$$

Clearly, by definition $P_{j}\left(\varepsilon, \eta_{1}, \ldots, \eta_{K}\right)=0$ for each $j$. Having such observation gives rise to a constructive scheme that exhibits the existence of a nontopological solution of the system (2) through finding a number $\varepsilon>0$ and functions $\eta_{1}, \ldots, \eta_{K}$ such that $P_{j}=0$ for $j=1, \ldots, K$ under the prescribed solutions of (31) and (37). Specifically, we take account of the mappings $P_{j}$ which are defined on $I \times D_{1}, j=1, \ldots, K$, where $I=\left(-\varepsilon_{0}, \varepsilon_{0}\right)$ and

$$
D_{1}=\left\{\eta=\left(\eta_{1}, \ldots, \eta_{K}\right) \in \prod_{K} Y_{\alpha}^{r}:\left\|\eta_{1}\right\|_{Y_{\alpha}}+\cdots+\left\|\eta_{K}\right\|_{Y_{\alpha}}<1\right\} .
$$

We remark that $P_{j}: I \times D_{1} \rightarrow X_{\alpha}^{r}$ is well-defined in the sense that it indeed maps into the space $X_{\alpha}^{r}$ with a given $\alpha \in(0,1)$ provided that $\varepsilon_{0}>0$ is chosen sufficiently small in terms of (35) and (38). Moreover, the singularity at $\varepsilon=0$ is removable; in fact, we define

$$
P_{j}(0, \eta)=\Delta \eta_{j}+\sum_{k=1}^{K} e^{v_{k}} \eta_{k}
$$

and by definition,

$$
\begin{aligned}
P_{j}(\varepsilon, \eta)-P_{j}(0, \eta)= & \varepsilon^{-2} \sum_{k=1}^{K} e^{v_{k}}\left[e^{\varepsilon^{2}\left(\eta_{k}+w\right)}-1-\varepsilon^{2}\left(\eta_{k}+w\right)\right] \\
& -\sum_{k=1}^{K} \sum_{m=1}^{K} e^{v_{k}+v_{m}}\left[e^{\varepsilon^{2}\left(\eta_{k}+\eta_{m}+2 w\right)}-1\right] .
\end{aligned}
$$

Therefore, $\left\|P_{j}(\varepsilon, \eta)-P_{j}(0, \eta)\right\|_{X_{\alpha}} \rightarrow 0$ as $\varepsilon \rightarrow 0$; in particular,

$$
P_{j}(0,0)=0, \quad j=1, \ldots, K .
$$

By a direct computation, the partial derivative $L$ of $P=\left(P_{1}, \ldots, P_{K}\right): I \times D_{1} \rightarrow \prod_{K} X_{\alpha}^{r}$ with respect to $\eta$ at $(\varepsilon, \eta)=$ $(0,0)$ is given by

$$
L:\left(\xi_{1}, \ldots, \xi_{K}\right) \mapsto\left(\Delta \xi_{1}+\sum_{m=1}^{K} e^{v_{m}} \xi_{m}, \ldots, \Delta \xi_{K}+\sum_{m=1}^{K} e^{v_{m}} \xi_{m}\right) .
$$

Lemma 3.1. The mapping $L: \prod_{K} Y_{\alpha}^{r} \rightarrow \prod_{K} X_{\alpha}^{r}$ is surjective.

Proof. Decompose $L=\Delta+A$, where $\Delta\left(\xi_{1}, \ldots, \xi_{K}\right)=\left(\Delta \xi_{1}, \ldots, \Delta \xi_{K}\right)$ and $A\left(\xi_{1}, \ldots, \xi_{K}\right)=\left(A_{1}, \ldots, A_{K}\right)$ with $A_{1}=\cdots=A_{K}=\sum_{m=1}^{K} e^{v_{m}} \xi_{m}$. We claim that $\Delta$ is bounded and $A$ is compact. In fact, from [3, Proposition 2.1] 
we see that the Laplace operator $\Delta$ is bounded. To prove the compactness of $A$, we let $\xi^{(j)}=\left(\xi_{1}^{(j)}, \ldots, \xi_{K}^{(j)}\right)$ be a bounded sequence in $\prod_{K} Y_{\alpha}^{r}$ such that

$$
\left\|\xi_{m}^{(j)}\right\|_{Y_{\alpha}} \leq M, \quad m=1, \ldots, K, j \in \mathbb{N},
$$

for some $M>0$. It suffices to show that $\left\{A_{1}\left(\xi^{(j)}\right)\right\}_{j=1}^{\infty}$ has a convergent subsequence. Let $B_{R}=\left\{x \in \mathbb{R}^{2}:|x|<R\right\}$ for $R_{1}>0$. Then

$$
\left\|\xi_{m}^{(j)}\right\|_{W^{2,2}\left(B_{2 R_{1}}\right)} \leq C\left\|\xi_{m}^{(j)}\right\|_{Y_{\alpha}} \leq C M, \quad \forall j, m .
$$

By the Rellich-Kondrachov Theorem [1], the imbedding $W^{2,2}\left(B_{2 R}\right) \hookrightarrow C^{1}\left(\overline{B_{R}}\right)$ is compact. We extract a subsequence $\xi^{(j)}$ and a continuous function $f=\left(f_{1}, \ldots, f_{K}\right)$ on $B_{R}$ such that

$$
\left\|\xi_{m}^{(j)}-f_{m}\right\|_{L^{\infty}\left(B_{R_{1}}\right)} \rightarrow 0, \quad m=1, \ldots, K
$$

as $j \rightarrow+\infty$. So that $A_{1}\left(\xi^{(j)}\right) \rightarrow A_{1}(f)$ pointwise on $B_{R}$. Repeat the processes on $B_{R_{k}}$ where $R_{k} \rightarrow+\infty$ as $k \rightarrow$ $+\infty$. We extend $f$ to be an entire function such that $A_{1}\left(\xi^{(j)}\right) \rightarrow A_{1}(f)$ pointwise on $\mathbb{R}^{2}$ by passing a subsequence. Furthermore, since $\omega>2 N_{1}+2$, we may pick $\sigma>0$ such that

$$
e^{v_{m}(r)}=O\left(r^{-2-\sigma}\right) \quad \text { as } r \rightarrow+\infty
$$

for all $m=1, \ldots K$. Choose $0<\alpha<\min \{1, \sigma\}$. Applying Proposition 3.1 gives

$$
\begin{aligned}
\left(1+|\mathbf{x}|^{2+\alpha}\right) A_{1}^{2}\left(\xi_{m}^{(j)}\right) & \leq c_{1}\left(1+|\mathbf{x}|^{2+\alpha}\right)\left(\sum_{m=1}^{K} e^{v_{m}} \xi_{m}^{(j)}\right)^{2} \\
& \leq c_{2}(1+|\mathbf{x}|)^{-2-2 \sigma+\alpha} \sum_{m=1}^{K}\left|\xi_{m}^{(j)}\right|^{2} \\
& \leq c_{3}(1+|\mathbf{x}|)^{-2-2 \sigma+\alpha} \log ^{2}(1+|\mathbf{x}|) \\
& \leq c_{4}(1+|\mathbf{x}|)^{-2-\sigma}
\end{aligned}
$$

for all $j$. By the dominated convergence theorem, $\left\|A_{1}\left(\xi_{m}^{(j)}\right)-A_{1}(f)\right\|_{X_{\alpha}} \rightarrow 0$ as $j \rightarrow+\infty$. Therefore, the operator $A$ is compact. So we conclude that the image of $L$, denoted by $\operatorname{Im} L$, is closed in the space $\prod_{K} X_{\alpha}^{r}$; please see [3, Proposition 2.1] or [10, Lemma 5.1]. Accordingly, $\prod_{K} X_{\alpha}^{r}=\operatorname{Im} L \oplus(\operatorname{Im} L)^{\perp}$. Suppose $L$ is not surjective. There can be a nonzero element $\zeta \in \prod_{K} X_{\alpha}^{r}$ such that $\langle\zeta, L y\rangle_{X_{\alpha}^{K}}^{K}=0$, i.e.

$$
\sum_{k=1}^{K} \int_{\mathbb{R}^{2}}\left(\psi_{k} \Delta y_{k}+\psi_{k} \sum_{m=1}^{K} e^{v_{m}} y_{m}\right) d \mathbf{x}=0 \quad \text { for all } y \in \prod_{K} Y_{\alpha}^{r},
$$

where $\psi_{k}=\zeta_{k}\left(1+|\mathbf{x}|^{2+\alpha}\right)$. Note that the space $Y_{\alpha}$ contains $C_{0}^{\infty}\left(\mathbb{R}^{2}\right)$, the set of all smooth functions with compact support. By elliptic regularity $\psi_{k}$ is a $C^{2}$-function for $k=1, \ldots, K$. Hence applying integration by parts on (41) gives

$$
\Delta \psi_{k}+e^{v_{k}} \sum_{m=1}^{K} \psi_{m}=0, \quad k=1, \ldots, K .
$$

Moreover, by definition,

$$
\int_{\mathbb{R}^{2}} \psi_{k}^{2}\left(1+|\mathbf{x}|^{2+\alpha}\right)^{-1} d \mathbf{x}=\left\|\zeta_{k}\right\|_{X_{\alpha}}^{2}<+\infty
$$

which indicates that each $\psi_{k} \in Y_{\alpha}^{r}$. Let $\Psi=\sum_{m=1}^{K} \psi_{m}$. By (42),

$$
\Delta \Psi+\left(\sum_{k=1}^{K} e^{v_{k}}\right) \Psi=0
$$


We have two cases in the following:

i) If $\Psi \equiv 0$, then from (42) we conclude that $\psi_{k} \equiv c_{k}$ where $c_{k}$ is a constant for each $k$. Note that $\left|c_{1}\right|+\cdots+\left|c_{K}\right| \neq 0$. We assume $c_{1} \neq 0$. Taking $y=(\xi, 0, \ldots, 0)$ in (41) implies that

$$
\int_{\mathbb{R}^{2}} c_{1} \Delta \xi=0 \text { for all } \xi \in Y_{\alpha}^{r},
$$

which is evidently impossible.

ii) If $\Psi$ is nontrivial, $\Psi(0) \neq 0$. We assume $\Psi(0)>0$. Then $\Psi$ is positive in an interval $(0,2 \rho)$ for some $\rho>0$. Let $\lambda(r)$ be a smooth cut-off function satisfying $\lambda(r)=1,0 \leq r \leq \rho$, and $\lambda(r)=0$ for $r \geq 2 \rho$. Let $\chi$ solve the equation

$$
\Delta \chi+\left(\sum_{k=1}^{K} e^{v_{k}}\right) \chi=\lambda \Psi .
$$

Note that $\chi \in Y_{\alpha}^{r}$ and considering $y=(\chi, \ldots, \chi)$ in (41) it follows that

$$
0=\int_{\mathbb{R}^{2}} \Psi \Delta \chi+\Psi\left(\sum_{k=1}^{K} e^{v_{k}}\right) \chi d \mathbf{x}=\int_{|\mathbf{x}|<2 \rho} \lambda|\Psi|^{2} d \mathbf{x}>0
$$

this is a contradiction.

Therefore, $\operatorname{Im} L=\prod_{K} X_{\alpha}^{r}$.

Proof of Theorem 3.1. Applying the Implicit Function Theorem [11, Theorem 2.7.5] on the mapping $P(\varepsilon, \eta)$, it is possible to represent $\eta$ in terms of $\varepsilon$ in an interval $\left(-\varepsilon_{1}, \varepsilon_{1}\right)$ for a small $\varepsilon_{1}>0$ and

$$
P\left(\varepsilon, \eta_{1}^{\varepsilon}, \eta_{2}^{\varepsilon}, \ldots, \eta_{K}^{\varepsilon}\right)=0 \text { for all } \varepsilon \in\left(-\varepsilon_{1}, \varepsilon_{1}\right) \text {. }
$$

Giving this back with the substitution (36), we obtain a family of nontopological solutions $\left(u_{1}^{\varepsilon}, u_{2}^{\varepsilon}, \ldots, u_{K}^{\varepsilon}\right)$ of the system (2), being dependent on $\varepsilon$ and having the expression

$$
u_{j}^{\varepsilon}(r)=v_{j}(\varepsilon r)+\varepsilon^{2} w(\varepsilon r)+\varepsilon^{2} \eta_{j}^{\varepsilon}(\varepsilon r)+2 \log \varepsilon
$$

for $j=1,2, \ldots, K$. Note that the solution $v_{j}$ of (31) is invariant under the scaling that

$$
v_{j}^{\varepsilon}(r)=v_{j}(\varepsilon r)+2 \log \varepsilon, \quad j=1,2, \ldots, K,
$$

and thus $\alpha$ is independent of $\varepsilon$. By virtue of (43) with the substitution $u_{j}=u_{j}^{\varepsilon}$ into the system (2), we have

$$
\begin{aligned}
\int_{0}^{\infty} r\left(\sum_{j=1}^{K} e^{u_{j}}\right)\left(1-\sum_{j=1}^{K} e^{u_{j}}\right) d r & =2 N_{1}-\lim _{r \rightarrow+\infty} r u_{1}^{\prime}(r) \\
& =\omega-\varepsilon^{2} \lim _{s \rightarrow+\infty} s w^{\prime}(s)-\varepsilon^{2} \lim _{s \rightarrow+\infty} s\left(\eta_{1}^{\varepsilon}\right)^{\prime}(s) \\
& =\omega+o(\varepsilon),
\end{aligned}
$$

as $\varepsilon \rightarrow 0$, where we use the facts that the limit

$$
\lim _{s \rightarrow+\infty} s w^{\prime}(s)=\int_{0}^{\infty} r\left[\left(\sum_{j=1}^{K} e^{v_{j}}\right)^{2}-\left(\sum_{j=1}^{K} e^{v_{j}}\right) w\right] d r
$$

converges; on the other hand, since $X_{\alpha} \hookrightarrow L^{1}\left(\mathbb{R}^{2}\right)$, 


$$
\lim _{s \rightarrow+\infty} s\left(\eta_{1}^{\varepsilon}\right)^{\prime}(s)=\int_{0}^{\infty} r \Delta \eta_{1}^{\varepsilon} d r=\frac{1}{2 \pi}\left\|\Delta \eta_{1}^{\varepsilon}\right\|_{L^{1}\left(\mathbb{R}^{2}\right)} \leq C\left\|\Delta \eta_{1}^{\varepsilon}\right\|_{X_{\alpha}} \leq C\left\|\eta_{1}^{\varepsilon}\right\|_{Y_{\alpha}} .
$$

Therefore, $\beta_{\varepsilon} \rightarrow \omega$ as $\varepsilon \rightarrow 0$.

\section{Sharp lower bound of flux for $N_{1}>2 N_{K}+1$}

In this section, we are interested in studying the following initial value problem

$$
\left\{\begin{array}{l}
v^{\prime \prime}(r)+\frac{1}{r} v^{\prime}(r)+f(r) e^{v(r)}\left[1-f(r) e^{v(r)}\right]=0, \quad r>0, \\
v(1)=\theta, \quad v^{\prime}(1)=\eta,
\end{array}\right.
$$

where $\theta, \eta \in \mathbb{R}$ are given initial data, it will be shown that solutions of (44) can be categorized into various types introduced in Definition 4.1.

Definition 4.1. Any solution $v(r)$ of (44) is classified as follows according to its behavior as $r \rightarrow 0$.

Type R-*: $v(r)$ is regular at 0 , i.e., $v(r)$ converges to a constant as $r \rightarrow 0$.

Type $\mathrm{P}-*: v(r)$ is positively singular at 0 , i.e., $v(r) \rightarrow+\infty$ as $r \rightarrow 0$.

Type $\mathrm{N}-*: v(r)$ is negatively singular at 0 , i.e., $v(r) \rightarrow-\infty$ as $r \rightarrow 0$.

To achieve our goal, we introduce the following initial value problems:

$$
\begin{aligned}
& \left\{\begin{array}{l}
\left\{r V_{0}^{\prime}(r)\right\}^{\prime}+r f(r) e^{V_{0}(r)}\left[1-f(r) e^{V_{0}(r)}\right]=0, \quad r>0, \\
V_{0}(0)=a,
\end{array}\right. \\
& \left\{\begin{array}{l}
\left\{r V_{\infty}^{\prime}(r)\right\}^{\prime}+r f(r) e^{V_{\infty}(r)}\left[1-f(r) e^{V_{\infty}(r)}\right]=0, \quad r>0, \\
\lim _{r \rightarrow \infty}\left[V_{\infty}(r)+4\left(N_{1}-N_{k}\right) \log r\right]=b,
\end{array}\right.
\end{aligned}
$$

where $a, b \in \mathbb{R}$. By $N_{K} \geq 0$ and $N_{1}>2 N_{K}+1$, we denote the unique solutions of (45) and (46) by $V_{0}(r ; a)$ and $V_{\infty}(r ; b)$, respectively. We note that from (44) and (45), $r V_{0}^{\prime}(r) \rightarrow 0$ as $r \rightarrow 0$ and

$$
r V_{0}^{\prime}(r)=-\int_{0}^{r} s f(s) e^{V_{0}(s)}\left[1-f(s) e^{V_{0}(s)}\right] d s, \quad r>0
$$

since $N_{1} \geq 0$. Define

$$
\gamma_{1}(a)=\left(V_{0}(1 ; a), V_{0}^{\prime}(1 ; a)\right) \quad \text { and } \quad \gamma_{2}(b)=\left(V_{\infty}(1 ; b), V_{\infty}^{\prime}(1 ; b)\right)
$$

for $a, b \in \mathbb{R}$, and let $\Gamma_{1}$ and $\Gamma_{2}$ be the ranges of $\gamma_{1}$ and $\gamma_{2}$ over $\mathbb{R}$, respectively. We note that both $\gamma_{1}$ and $\gamma_{2}$ are smooth by the assumptions $N_{K} \geq 0$ and $N_{1}>2 N_{K}+1$ again. In fact, $\Gamma_{1}$ and $\Gamma_{2}$ are the collections of initial data corresponding to solutions of Type $\mathrm{R}-*$ and $*-\mathrm{R}$ for (44), respectively, where definition of Type $*-\mathrm{R}$ be described as follows:

Type $*$-R: $\frac{v(r)}{4\left(N_{1}-N_{K}\right) \log r}$ converges to -1 as $r \rightarrow \infty$.

We now present some facts, stated in Lemma 4.1 below, which are involving the characterization of solutions of various types in terms of $\widetilde{E}_{L}(r ; v)$, where $\widetilde{E}_{L}(r ; v)=E(r ; v)+L r v^{\prime}(r)$ if $4 N_{K}>L>2 N_{K}>0$ and $\widetilde{E}_{L}(r ; v)=$ $E(r ; v)$ if $N_{K}=0$.

Lemma 4.1. Suppose $v(r)$ is a solution of (44), then the following assertions are true.

(a) If $v(r)$ is of Type $R-*$, then $\widetilde{E}_{L}(r ; v) \rightarrow 0$ as $r \rightarrow 0^{+}$. 
(b) If $v(r)$ is of Type $P-*$, then $\widetilde{E}_{L}(r ; v) \rightarrow C$ for some $C<0$ as $r \rightarrow 0^{+}$.

(c) If $v(r)$ is of Type $N-*$, then $\widetilde{E}_{L}(r ; v) \rightarrow C$ for some $C>0$ as $r \rightarrow 0^{+}$.

Proof. By the proof of Lemma 2.1, we see that $f e^{v(r)}<1$ and $\left[1-f e^{v(r)}\right]^{\prime}<0$ on $\left[0, r_{0}\right)$ for some $r_{0}>0$. Then it is not difficult to obtain these results (a)-(c), and hence we omit the proof.

Proposition 4.2. The following assertions on the solution $v(r ; \theta, \eta)$ of $(44)$ are true.

(i) If $v\left(r ; \theta_{0}, \eta_{0}\right)$ is of Type $P-*$, then there exists $\delta>0$ such that $v(r ; \theta, \eta)$ is of Type $P-*$ for $(\theta, \eta) \in B_{\delta}\left(\left(\theta_{0}, \eta_{0}\right)\right)$.

(ii) If $v\left(r ; \theta_{0}, \eta_{0}\right)$ is of Type $N$-*, then there exists $\delta>0$ such that $v(r ; \theta, \eta)$ is of Type $N$-* for $(\theta, \eta) \in B_{\delta}\left(\left(\theta_{0}, \eta_{0}\right)\right)$.

Proof. (i) On the contrary. We may assume that there exists a sequence $\left\{\left(\theta_{n}, \eta_{n}\right)\right\}_{n \in N}$ such that $\left(\theta_{n}, \eta_{n}\right) \rightarrow\left(\theta_{0}, \eta_{0}\right)$ as $n \rightarrow \infty$ and $v\left(r ; \theta_{n}, \eta_{n}\right)$ is a solution of Type $\mathrm{R}-*$ or Type $\mathrm{N}-*$ for all $n \in N$. Since $v\left(r ; \theta_{0}, \eta_{0}\right)$ is a solution of Type P-* and Lemma 4.1, there exist two constants $R_{0}, \delta>0$ such that $v^{\prime}\left(R_{0} ; \theta, \eta\right)<0, \widetilde{E}_{L}\left(R_{0} ; v\left(R_{0} ; \theta, \eta\right)\right)<0$ and

$$
\frac{\partial \widetilde{E}_{L}}{\partial r}(r ; v(r ; \theta, \eta))=\left[2 \frac{r f^{\prime}(r)}{f(r)}-L\right] r f e^{v(r ; \theta, \eta)}\left[1-f e^{v(r ; \theta, \eta)}\right]+2 r f e^{v(r ; \theta, \eta)}\left[2-f e^{v(r ; \theta, \eta)}\right] \geq 0
$$

on $\left[0, R_{0}\right]$ for all $\left|(\theta, \eta)-\left(\theta_{0}, \eta_{0}\right)\right|<\delta$. Consequently, there exists a sequence $\left\{r_{n}\right\}_{n \in N}$ such that $r_{n}$ be the first local maximum point of $v\left(r ; \theta_{n}, \eta_{n}\right)$ on $\left[0, R_{0}\right], v^{\prime}\left(r_{n} ; \theta_{n}, \eta_{n}\right)=0$ and $r_{n} \rightarrow 0$ as $n \rightarrow \infty$. Then we have $\widetilde{E}_{L}\left(r ; v\left(r ; \theta_{n}, \eta_{n}\right)\right)>0$ on $\left[r_{n}, R_{0}\right]$ for large $n$ by $\widetilde{E}_{L}\left(r_{n} ; v\left(r_{n} ; \theta_{n}, \eta_{n}\right)\right)=0$ and $\frac{\partial \widetilde{E}_{L}}{\partial r}\left(r ; v\left(r ; \theta_{n}, \eta_{n}\right)\right) \geq 0$ on $\left[0, R_{0}\right]$ for large $n$. It is a contradiction to $\widetilde{E}_{L}\left(R_{0} ; v\left(R_{0} ; \theta_{0}, \eta_{0}\right)\right)<0$ and hence we finish the result (i).

(ii) Since $v\left(r ; \theta_{0}, \eta_{0}\right)$ is of Type $\mathrm{N}-*$, there exist two constants $\delta>0$ and $R_{0}>0$ such that

$$
v^{\prime}\left(R_{0} ; \theta, \eta\right)>0 \quad \text { and }\left[1-f e^{v\left(R_{0} ; \theta, \eta\right)}\right]<0 \quad \text { for all }\left|(\theta, \eta)-\left(\theta_{0}, \eta_{0}\right)\right|<\delta .
$$

Then, by the proof of Lemma 2.1, we have $\left(r v^{\prime}\right)^{\prime}(r ; \theta, \eta)=r f e^{v(r ; \theta, \eta)}\left[f e^{v(r ; \theta, \eta)}-1\right] \leq 0$ on $\left(0, R_{0}\right)$ for any $\mid(\theta, \eta)-$ $\left(\theta_{0}, \eta_{0}\right) \mid<\delta$ and hence we finish the result (ii).

Theorem 4.1. If $N_{1}>2 N_{K}+1$, then there exists a constant $s_{0}=s_{0}\left(N_{1}, \ldots, N_{K}\right) \in \mathbb{R}$ such that $\beta\left(s_{0}\right)=\min \{\beta(s)$ : $s \in \mathbb{R}\}$ and $\beta\left(s_{0}\right) \in\left(2 N_{1}+2,4\left(N_{1}-N_{K}\right)\right)$. Moreover, Eq. (14) is solvable for any prescribed value $\beta \in\left[\beta\left(s_{0}\right)\right.$, $\left.4 N_{1}+4\right)$.

Proof. Since $N_{1}>2 N_{K}+1$, there exists constant $\delta<0$ such that $N_{1}+2 \delta>2 N_{K}+1$. Let $\widetilde{w}(s)$ satisfy

$$
\left\{\begin{array}{l}
\left\{s \widetilde{w}^{\prime}(s)\right\}^{\prime}+s \widetilde{K}(s) e^{\widetilde{w}(s)}=0, \quad s \geq 0 \\
\widetilde{w}(0)=c \in \mathbb{R}, \quad \widetilde{w}^{\prime}(0)=0
\end{array}\right.
$$

where $\widetilde{K}(s)=s^{\sigma-4} f\left(\frac{1}{s}\right)$ and $\sigma=4\left(N_{1}-N_{K}+\delta\right)$. It is easy to see that

$$
\widetilde{K}(s)=f_{\infty} s^{2 \widetilde{p}} \quad \text { near } s=0 \quad \text { and } \quad \widetilde{K}(s)=f_{0} s^{2 \widetilde{q}} \quad \text { near } s=\infty,
$$

where

$$
\left\{\begin{array}{l}
(\tilde{p}, \widetilde{q})=\left(N_{1}-2 N_{K}-2+2 \delta, 2 N_{1}-3 N_{K}-2+2 \delta\right), \\
\left(f_{0}, f_{\infty}\right)=\left(\lim _{r \rightarrow \infty} r^{-2 N_{1}} f(r), \lim _{r \rightarrow 0} r^{-2 N_{K}} f(r)\right) .
\end{array}\right.
$$

To prove this theorem, we need the following fact.

Claim. (47) possesses a solution $\widetilde{w}(s)$ with $\lim _{s \rightarrow \infty} \frac{\widetilde{w}(s)}{\log s}=-4(1+\widetilde{q}-\delta)$.

Proof of the claim. According to [6] and $\widetilde{q}>2 \widetilde{p}+1$, we obtain that

$$
\lim _{c \rightarrow \infty} \gamma(s)=4(\tilde{p}-\widetilde{q}) \text { and } \lim _{c \rightarrow-\infty} \gamma(s)=-4(1+\widetilde{q}),
$$

where $\widetilde{w}(s ; c)=\gamma(c) \log s+O(1)$ as $s \rightarrow \infty$. Thus we complete this claim. 
Combining with the similarly argument of the proof in Theorem 3.1 and claim, we obtain that there exists a function $z_{0}(s)$ in $C^{2}[0, \infty)$ which satisfies $\lim _{s \rightarrow \infty} \frac{z_{0}(s)}{\log s}=-4(1+\tilde{q}-\delta)$ and

$$
\left\{\begin{array}{l}
\left\{s z_{0}^{\prime}(s)\right\}^{\prime}+s \widetilde{K}(s) e^{z_{0}(s)}\left[1-s^{4} \widetilde{K}(s) e^{z_{0}(s)}\right]=0, \quad s \geq 0, \\
z_{0}(0) \in \mathbb{R}, \quad z_{0}^{\prime}(0)=0 .
\end{array}\right.
$$

According to [6] and $N_{1}>2 N_{k}+1$, we have

$$
\lim _{a \rightarrow-\infty} \gamma_{1}(a)=(-\infty, 0), \quad \lim _{b \rightarrow+\infty} \gamma_{2}(b)=\left(-\infty, 4\left(-1-N_{K}\right)\right)
$$

Moreover, by Lemma 2.3 and Proposition 4.2, we get that there exists a constant $a_{0} \in J_{\infty}$ such that for all $a \geq a_{0}$, $\gamma_{1}(a)$ lie on the first quadrant which satisfies

$$
\lim _{a \rightarrow a_{1}}\left|\gamma_{1}(a)\right|=\infty \quad \text { for some } a_{1} \in\left(a_{0}, \infty\right]
$$

and $v(r ;-\log f(1), \eta)$ be of Type $\mathrm{N}-*$ for large $\eta>0$. From (48), (49) and Proposition 4.2, we conclude that $v(r ; \theta, \eta)$ is of Type $\mathrm{N}-*$ (resp., Type P-*) if $(\theta, \eta)$ lies on the same side (resp., the opposite side) of $\eta^{+}$-axis with respect to $\Gamma_{1}$. Now we let $\left(r, v_{0}(r)\right)=\left(s^{-1}, z_{0}(s)+\sigma \log s\right)$, and hence $v_{0}(r)$ be a solution of Type N-R for (44). Then $\Gamma_{1}$ and $\Gamma_{2}$ have an intersection point $\left(\theta_{0}, \eta_{0}\right)$ in $\theta \eta$-plan due to $1+N_{K}>0$ and (48). Hence (14) possesses a solution $v_{0}(r)$ such that

$$
\int_{0}^{\infty} r f(r) e^{v_{0}(r)}\left[1-f(r) e^{v_{0}(r)}\right] d r<4\left(N_{1}-N_{K}\right) .
$$

Combining with $\lim _{s \rightarrow-\infty} \beta(s)=4\left(N_{1}-N_{K}\right)$ and Theorem 2.2, there exists an $s_{0} \in \mathbb{R}$ such that

$$
\beta\left(s_{0}\right)=\min \{\beta(s): s \in \mathbb{R}\} \quad \text { and } \quad \beta\left(s_{0}\right) \in\left(2 N_{1}+2,4\left(N_{1}-N_{K}\right)\right) .
$$

Therefore, we complete the proof of Theorem 4.1.

\section{Conflict of interest statement}

The authors certify that they have NO affiliations with or involvement in any organization or entity with any financial interest in the subject matter or materials discussed in this manuscript.

\section{Acknowledgement}

The authors would like to express sincere thank to the anonymous referee whose carefully reading the manuscript and valuable comments greatly improve the manuscript.

\section{Appendix A}

In the appendix, we will prove the radial symmetry of the topological solutions for (2). Without loss of generality, we also assume that $N=N_{1} \geq N_{i}$ for $i=2, \ldots, K$ and let $(u(x), P(r))=\left(u_{1}(x), 1+\sum_{i=2}^{K} L_{i} r^{2\left(N_{i}-N_{1}\right)}\right)$ where $L_{i}=e^{\alpha_{1}-\alpha_{i}}$ and $r=|x|$. However, it is easy to see that if we want to discuss the couple equations (2), then we only consider the following equation:

$$
\Delta u(x)+P(|x|)\left(1-P(|x|) e^{u(x)}\right) e^{u(x)}=4 \pi N \delta_{0} \quad \text { in } \mathbb{R}^{2} .
$$

We give a result about the radially symmetric property of topological solution as follows.

Theorem A.1. All topological solutions $\left(u_{1}(x), \ldots, u_{K}(x)\right)$ of (2) are radially symmetric, that is, all solutions $u(x)$ of (A.1) which satisfy $\lim _{|x| \rightarrow \infty} u(x)=C_{u}$ are radially symmetric where $C_{u}=-\log \lim _{|x| \rightarrow \infty} P(|x|)$. 
Proof. We may assume $P(\infty)=\lim _{|x| \rightarrow \infty} P(|x|)=1$ from scaling method with

$$
(\hat{u}(x), \hat{P}(|x|))=\left(u(x)-C_{u}, e^{C_{u}} P(|x|)\right) .
$$

Combining maximum principle and $P(|x|)>1$ for $|x|>0$, we see that $u(x)$ does not have nonnegative local maximum value on $\mathbb{R}^{2} \backslash\{O\}$. Hence we obtain that $u(x)<0$ for $|x|>0$. Now we want to apply the method of moving plane with some modifications to prove that $u(x)$ is radially symmetric on $\mathbb{R}^{2}$. For $0<\sigma<R$, we define the sets $\Sigma_{\sigma}=\left\{x \in \mathbb{R}^{2}: x_{1}>\sigma\right\}, T_{\sigma}=\left\{x \in \mathbb{R}^{2}: x_{1}=\sigma\right\}$, and $u_{\sigma}(x)=u\left(x^{\sigma}\right)$ for $x \in \Sigma_{\sigma}$, where $x^{\sigma}$ is the reflection of $x$ with respect to the line $x_{1}=\sigma$, i.e., $x^{\sigma}=\left(2 \sigma-x_{1}, x_{2}\right)$. Set $w_{\sigma}(x)=u(x)-u_{\sigma}(x)$ for $x \in \Sigma_{\sigma}$. Define

$$
\left\{\begin{array}{l}
S_{u}=\left\{\rho \in(0, \infty): w_{\sigma}>0 \text { in } \Sigma_{\sigma} \text { for } \sigma \in(\rho, \infty)\right\}, \\
\rho_{u}=\inf _{\rho \in S_{u}}\{\rho\} .
\end{array}\right.
$$

First, we show that $S_{u} \neq \emptyset$. On a contrary and maximum principle, we may assume that there exists a sequence $\left\{x_{k}\right\}_{k=1}^{\infty}$ such that

$$
\lim _{k \rightarrow \infty}\left|x_{k}\right|=\infty, \quad w_{k}\left(x_{k}\right)<0 \quad \text { and } \quad \Delta w_{k}\left(x_{k}\right) \geq 0 \quad \text { for all positive integer } k .
$$

However, by $\lim _{|x| \rightarrow \infty} u(x)=0, P^{\prime}(r)<0$ and $P(r)>1$ on $(0, \infty)$, we obtain that

$$
\begin{aligned}
\Delta w_{k}\left(x_{k}\right) & =P\left(\left|x_{k}^{k}\right|\right) e^{u_{k}}\left(1-P\left(\left|x_{k}^{k}\right|\right) e^{u_{k}}\right)-P\left(\left|x_{k}\right|\right) e^{u}\left(1-P\left(\left|x_{k}\right|\right) e^{u}\right) \\
& \leq P\left(\left|x_{k}\right|\right) e^{u_{k}}\left(1-P\left(\left|x_{k}\right|\right) e^{u_{k}}\right)-P\left(\left|x_{k}\right|\right) e^{u}\left(1-P\left(\left|x_{k}\right|\right) e^{u}\right) \\
& =-P\left(\left|x_{k}\right|\right) e^{\widetilde{u}\left(x_{k}\right)}\left(1-2 P\left(\left|x_{k}\right|\right) e^{\widetilde{u}\left(x_{k}\right)}\right) w_{k}\left(x_{k}\right)<0
\end{aligned}
$$

for large $k$ where $\widetilde{u}\left(x_{k}\right) \in\left[u\left(x_{k}\right), u_{k}\left(x_{k}\right)\right]$. It yields a contradiction to (A.3) and hence the set $S_{u}$ is nonempty. Next, we prove $\rho_{u}=0$. Suppose this is not true. Then, w.l.o.g., we can assume $\rho_{u}>0$. Then by continuity, we have $w_{\rho_{u}}(x) \geq 0$ in $\Sigma_{\rho_{u}}$. It is easy to see that $w_{\rho_{u}}$ satisfies the following equation

$$
\left\{\begin{array}{l}
\Delta w_{\rho_{u}}+C_{\rho_{u}}(x) w_{\rho_{u}}=-4 \pi N_{1} \delta\left(2 \rho_{u}, 0\right) \quad \text { in } \Sigma_{\rho_{u}}, \\
w_{\rho_{u}} \geq 0 \quad \text { in } \Sigma_{\rho_{u}} \cup T_{\rho_{u}}, \\
\lim _{|x| \rightarrow \infty} w_{\rho_{u}}(x)=0
\end{array}\right.
$$

where $\delta\left(2 \rho_{u}, 0\right)$ is the Dirac measure at the point $\left(2 \rho_{u}, 0\right)$ and

$$
C_{\rho_{u}}(x)=\frac{P(|x|) e^{u}\left(1-P(|x|) e^{u}\right)-P\left(\left|x^{\rho_{u}}\right|\right) e^{u_{\rho_{u}}}\left(1-P\left(\left|x^{\rho_{u}}\right|\right) e^{u_{\rho_{u}}}\right)}{u-u_{\rho_{u}}} \quad \text { for } x \in \Sigma_{\rho_{u}} .
$$

Thus, if $w_{\rho_{u}}\left(x_{1}\right)=0$ for some $x_{1} \in \Sigma_{\rho_{u}}$, then by (A.4) and maximum principle, we have $w_{\rho_{u}} \equiv 0$ in $\bar{\Sigma}_{\rho_{u}}$. However, this contradicts to the fact that $w_{\rho_{u}}\left(2 \rho_{u}, \varepsilon\right)=u\left(2 \rho_{u}, \varepsilon\right)-u(0, \varepsilon)>0$ for small $\varepsilon>0$. Therefore we obtain that

$$
\left\{\begin{array}{l}
w_{\rho_{u}}(x)>0 \text { for any } x \in \Sigma_{\rho_{u}} \\
w_{\rho_{u}}(x)=0 \text { on } T_{\rho_{u}} \\
\lim _{|x| \rightarrow \infty} w_{\rho_{u}}(x)=0
\end{array}\right.
$$

By (A.4)-(A.5) and Hopf Boundary Lemma, we obtain

$$
\frac{\partial w_{\rho_{u}}}{\partial x_{1}}>0 \text { on } T_{\rho_{u}} \text {. }
$$

On the other hand, since $\rho_{u}>0$, there exists a positive sequence $\varepsilon_{k}$ such that $\rho_{u}-\varepsilon_{k}>0$ and $\left(\rho_{u}-\varepsilon_{k}\right) \rightarrow \rho_{u}$ as $k \rightarrow \infty$. By the definition of $\rho_{u}$, for each $\varepsilon_{k}$, we obtain that $w_{\rho_{u}-\varepsilon_{k}}$ is non-positive somewhere in $\Sigma_{\rho_{u}-\varepsilon_{k}}$. By the way, we have $\lim _{|x| \rightarrow \infty} w_{\rho_{u}}(x)=0$ and $w_{\rho_{u}-\varepsilon_{k}}=0$ on $T_{\rho_{u}-\varepsilon_{k}}$. Hence, for each $\varepsilon_{k}$ there exists $x_{k} \in \Sigma_{\rho_{u}-\varepsilon_{k}}$ such that

$$
w_{\rho_{u}-\varepsilon_{k}}\left(x_{k}\right) \leq 0, \quad \nabla w_{\rho_{u}-\varepsilon_{k}}\left(x_{k}\right)=(0,0) \quad \text { and } \quad \Delta w_{\rho_{u}-\varepsilon_{k}}\left(x_{k}\right) \geq 0 .
$$


From the similarly argument of proof of $S_{u} \neq \phi$, we may assume that $\left\{x_{k}\right\}$ is a bounded sequence and there exists a convergent subsequence, we still denote it by $x_{k}$, such that $x_{k} \rightarrow x_{0}$. By (A.7) we obtain that

$$
0 \geq \lim _{k \rightarrow \infty} w_{\rho_{u}-\varepsilon_{k}}\left(x_{k}\right)=w_{\rho_{u}}\left(x_{0}\right) .
$$

Hence, by the above inequality and (A.5), we conclude that $x_{0} \in T_{\rho_{u}}$ and, by (A.7),

$$
0=\lim _{k \rightarrow \infty} \frac{\partial w_{\rho_{u}-\varepsilon_{k}}}{\partial x_{1}}\left(x_{k}\right)=\frac{\partial w_{\rho_{u}}}{\partial x_{1}}\left(x_{0}\right) .
$$

This contradicts to (A.6), and hence $\rho_{u}=0$. Therefore, $u(x)$ is radially symmetric on $\mathbb{R}^{2}$.

\section{References}

[1] R.A. Adams, Sobolev Spaces, Academic Press, New York, 1975.

[2] D. Chae, Existence of the semilocal Chern-Simons vortices, J. Math. Phys. 46 (2005) 042303.

[3] D. Chae, O.Yu. Imanuvilov, The existence of non-topological multivortex solutions in the relativistic self-dual Chern-Simons theory, Commun. Math. Phys. 215 (2000) 119-142.

[4] H. Chan, C.C. Fu, C.-S. Lin, Non-topological multi-vortex solutions to the self-dual Chern-Simons-Higgs equation, Commun. Math. Phys. 231 (2002) 189-221.

[5] X. Chen, S. Hastings, J.B. McLeod, Y. Yang, A nonlinear elliptic equations arising from gauge field theory and cosmology, Proc. R. Soc. Lond. A 446 (1994) 453-478.

[6] K.S. Cheng, C.S. Lin, On the conformal Gaussian curvature equation in $\mathbb{R}^{2}$, J. Differ. Equ. 146 (1998) 226-250.

[7] G.W. Gibbons, M.E. Ortiz, F. Ruiz, T.M. Samols, Semilocal strings and monopoles, Nucl. Phys. B 385 (1992) 127-144.

[8] A. Khare, Semilocal self-dual Chern-Simons vertices, Phys. Rev. D 46 (1992) R2287-R2289.

[9] C.S. Lin, Uniqueness of solutions to the mean field equations for the spherical Onsager vortex, Arch. Ration. Mech. Anal. 153 (2000) $153-176$.

[10] A.V. Fursikov, O.Yu. Imanuvilov, Local exact boundary controllability of the Boussinesq equation, SIAM J. Control Optim. 36 (1998) $391-421$.

[11] L. Nirenberg, Topics in Nonlinear Analysis, Courant Lect. Notes Math., vol. 6, American Mathematical Society, 2001.

[12] D.H. Sattinger, Conformal metrics in $\mathbb{R}^{2}$ with prescribed curvature, Indiana Univ. Math. J. 22 (1972) 1-4.

[13] T. Vachaspati, A. Achucarro, Semilocal cosmic strings, Phys. Rev. D 44 (1991) 3067-3071. 